\title{
THE PRINCIPAL TYPE-SCHEME OF AN OBJECT IN COMBINATORY LOGIC
}

\author{
BY \\ R. HINDLEY
}

Introduction. In their book Combinatory Logic [1], Curry and Feys introduced the notion of "functional character" (here called "type-scheme") of an object of combinatory logic. Roughly speaking, each object of combinatory logic ("ob" for short) represents a function or an operator on functions; for instance the ob $\mathbf{I}$ represents the identity operator, and we have for all obs $X$,

$$
I X=X \text {. }
$$

One of the aims of combinatory logic is to study the most basic properties of functions and other concepts, with as few restrictions as possible; hence in the simplest form of combinatory logic there is nothing to stop a function being applied to itself; thus $X X$ is an ob for all obs $X$. However it is also natural to look at the consequences of introducing type-restrictions, formulated by assigning types to the obs according to certain rules, to be described later.

Each type is a linguistic expression representing a set of functions, and for any type $\alpha$ the statement " $X$ has type $\alpha$ " is intended to mean that the ob $X$ represents a member of the set represented by $\alpha$. Given types $\alpha$ and $\beta$, the set of all functions from the set $\alpha$ into the set $\beta$ is represented by the type "F $\alpha \beta$ " (using Curry's notation). Now consider the ob I; if $X$ has type $\alpha$, then the ob $\mathbf{I} X$ must also have type $\alpha$. Hence I represents a function from $\alpha$ into $\alpha$, and so it must be given the type $F_{\alpha \alpha}$, for each type $\alpha$. Thus $I$ has not just one type, but a whole class of types. This might seem strange, but it comes from the fact that I represents the abstract notion of "identity-function", rather than one particular identity-function for a particular set. The identity-function involves basically the same concept, no matter what we are applying it to. Similarly the other two basic combinators $\mathbf{S}$ and $\mathrm{K}$ (see later, or $[1, \S 5 \mathrm{~A}]$ ) represent certain simple operations which can be performed on almost any functions, and thus they too have an infinite class of types (see $\$ 1$ later for details, and $\S 5$ for comment).

To denote classes of types, we can use variables $a, b, c$; then the fact that I has every type of form $\mathbf{F} \alpha \alpha$ can be expressed by assigning to I the type-scheme

Faa.

Received by the editors February 22, 1969. 
We shall see later that all the types which the rules assign to I have the form F $\alpha \alpha$, so the types of $\mathbf{I}$ are just those obtained by substituting a type $\alpha$ for the variables in the type-scheme Faa. It will not be obvious from the rules that every ob $X$ has a type-scheme with this property. If $X$ does have a type-scheme from which all the types of $X$ (and no extra types) can be obtained by substituting types for variables, it will be called a principal type-scheme of $X\left({ }^{1}\right)$.

In $\S 3$ of this paper it will be proved that every ob $X$ that has a type at all, has in fact a principal type-scheme which can be effectively determined from the structure of the ob. This result will apply to the case when $X$ may contain variables, which are not assigned types by the rules; in this case it will say that if there exists an assignment of types to the variables from which a type for $X$ can be deduced, then there is a type-scheme $\alpha$ such that all the types of $X$, for every assignment of types to the variables, are instances of $\alpha$.

$\$ 4$ will show how the principal type-scheme of $[x] . X$ (defined therein and in [1]) is related to the principal type-scheme of $X$.

Finally, in $\S 6$ it will be shown that if $\alpha$ is a principal type-scheme of an ob $X$, then any substitution-instance $\beta$ of $\alpha$ is a principal type-scheme of some ob $X_{\beta}$ (which is reducible to $X$ by the reduction-rules for combinators). Besides its intrinsic interest, this result goes part of the way towards justifying a conjecture of Curry's that the alternative system of combinatory logic with type-restrictions (in which an infinity of basic combinators is postulated, each with a unique type) can be defined in the system described here. This point will be explained in $\S 5$.

Actually, the main result (Theorem 1) in $\$ 3$ was proved independently, almost simultaneously, by Curry and myself, using different methods; the present exposition owes something to both. Curry's proof (see [2]) does not use the result of J. A. Robinson [4] which is needed here; in fact I think his proof essentially contains an alternative proof of that result. The main result in $\$ 4$ (Theorem 2) was also proved almost simultaneously by Curry and myself: since Curry's proof is shorter, it has been used here.

I wish to thank H. Curry for all his help with this paper, both in discussions and by permission to use his proof of Theorem 2 .

1. Definitions. The notions presented informally in the introduction will now be formalized. The symbol " $\equiv$ " will be used to denote identity of obs, types and type-schemes, since " =" will denote a defined equivalence relation (roughly, equality of interpretation) between obs. Obs will be regarded as linguistic expressions

(1) In [2] principal type-schemes are called "principal functional characters." In [1] the term "functional character" is used in approximately the sense of "principal type-scheme" here, but the assumptions therein allow that extra types could be assigned to the obs, besides the types given explicitly; if this were done the functional characters stated in [1] (e.g. \$8c, p. 264) would not be principal. 
intended to represent functions or more abstract operations (like $\mathbf{I}$ in the Introduction $)\left({ }^{2}\right)$.

Definition 1. Obs.

(i) There are assumed to be certain symbols called ob-atoms, all of which are obs; these atoms may include two basic combinators, $\mathbf{S}$ and $\mathbf{K}$.

(ii) If $X$ and $Y$ are obs, then the result, $(X Y)$, of putting $X$ and $Y$ in parentheses with $X$ on the left of $Y$, is a compound ob.

A combinator is any ob in which only the atoms $\mathbf{S}$ and $\mathbf{K}$ occur.

(Notice that this definition is really a sort of definition-scheme: for each given set of atoms, it defines by induction a corresponding set of obs. The exact nature of the atoms is irrelevant here (except that $\mathbf{S}$ and $\mathbf{K}$ will play a special role). Similarly Definitions 2, 4 and 5 are really definition-schemes.)

It is assumed that no compound ob is also an atom, and that if $(X Y) \equiv(U V)$, then $X \equiv Y$ and $U \equiv V$. Capital Roman letters denote arbitrary obs, and parentheses will be omitted in such a way that for instance, " $X Y Z U$ " denotes $(((X Y) Z) U)$.

Definition 2. Types and Type-schemes.

(i) There are assumed to be certain symbols called basic types (at least one and at most countably many); also a countably infinite sequence of type-rariables. All these are type-schemes, called type-atoms.

(ii) If $\alpha$ and $\beta$ are type-schemes, then (F $\alpha \beta)$ is a compound type-scheme.

A type is any type-scheme which contains no variables $\left({ }^{3}\right)$.

It is assumed that no compound type-scheme is a type-atom and that if $\left(F_{\alpha \beta}\right)$ $\equiv(\mathbf{F} \gamma \delta)$ then $\alpha \equiv \beta$ and $\gamma \equiv \delta$. Greek letters denote arbitrary type-schemes, and lower case Roman letters from the start of the alphabet denote type-variables. The outermost pair of parentheses on a type-scheme will usually be omitted. In denoting lists of variables, for example " $a_{1}, \ldots, a_{n}$ ", it will be assumed that the variables are mutually distinct (more precisely, $i \neq j$ implies $a_{i} \not \equiv a_{j}$ ), unless explicitly stated otherwise.

In the usual interpretations, the ob $(X Y)$ represents the result of applying the function $X$ to the argument $Y$. Each basic type represents a particular set of functions, depending on the interpretation one may have in mind, and $F_{\alpha} \beta$ represents the set of all functions from $\alpha$ into $\beta$. Thus each type represents a particular set of functions.

The variables are intended to represent arbitrary types, so that a type-scheme $\alpha$ containing variables represents a class of types, each type being obtained from $\alpha$ by substituting types for the variables.

${ }^{(2)}$ In Curry [1] the obs are not necessarily linguistic expressions, but it is convenient to think of them here as such, and this specialization involves no essential loss of generality. But in Definition 1, notice for example that the symbols " $\mathbf{S}$ " and " $K$ " need not themselves be the basic combinators; they only denote them.

${ }^{3}$ ) In [1], Curry finds it convenient to regard types as a particular kind of ob, called " $F$-obs"; the results of the present paper apply to this case. 
Sequences will be denoted by angle brackets, for example " $\left\langle\alpha_{1}, \ldots, \alpha_{n}\right\rangle$ ", and sets denoted by brackets " \{\} ".

Definition 3. Substitution. For any type-schemes $\beta_{1}, \ldots, \beta_{n}, \alpha$, and distinct type-variables $a_{1}, \ldots, a_{n}$,

$$
\left[\frac{\beta_{1}, \ldots, \beta_{n}}{a_{1}, \ldots, a_{n}}\right] \alpha
$$

is defined to be the result of replacing each occurrence of $a_{i}$ in $\alpha$ by $\beta_{i}$, simultaneously for $i=1 \cdots n$. (If $a_{i}$ does not occur in $\alpha$, no replacements are made.) Any type-scheme with the above form is called an instance of $\alpha$. (Hence $\alpha$ is an instance of itself.) For any sequence $\left\langle\alpha_{1}, \ldots, \alpha_{k}\right\rangle$ of type-schemes, define

$$
\left[\frac{\beta_{1}, \ldots, \beta_{n}}{a_{1}, \ldots, a_{n}}\right]\left\langle\alpha_{1}, \ldots, \alpha_{k}\right\rangle
$$

to be the sequence formed by substituting $\beta_{i}$ for $a_{i}$ in all of $\alpha_{1}, \ldots, \alpha_{k}$, simultaneously for $i=1, \ldots, n$.

An expression consisting of a type-scheme $\alpha$ beside an ob $X$ is called a statement, $\alpha X$. The statement $\beta X$ is called an instance of $\alpha X$ iff $\beta$ is an instance of $\alpha$.

Definition 4. Type-schemes of obs. The type-scheme $\alpha$ is a type-scheme of $X$ iff the statement $\alpha X$ can be deduced from the axioms below by the rule (F) below.

Axiom-schemes. Each axiom-scheme is assumed to be a statement $\beta Y$, where $Y$ is an ob-atom, and no two axiom-schemes contain the same $Y$. If the ob-atoms include $\mathbf{S}$ and $\mathbf{K}$, then the axiom-schemes include

$$
\begin{aligned}
& \mathbf{F}(\mathbf{F} a(\mathbf{F} b c))(\mathbf{F}(\mathbf{F} a b)(\mathbf{F} a c)) \mathbf{S} \\
& \mathbf{F} a(\mathbf{F} b a) \mathbf{K}
\end{aligned}
$$

for some (distinct) type-variables $a, b, c$. The axioms are all the instances of the axiom-schemes. (Including the schemes themselves; this is just a notational convenience.)

Rule (F). From $\left(F_{\gamma} \delta\right) X$ and $\gamma Y$, deduce $\delta(X Y)$.

If $\alpha$ contains no variables, and $\alpha X$ is deduced from axioms containing no variables, we say that $\alpha$ is a type of $X$.

Definition 5. Deductions. Given a set $\mathscr{A}$ of statements, a deduction of $\alpha X$ from $\mathscr{A}$ is a finite set of statements arranged as a tree in the usual way, with $\alpha X$ at the bottom. Each statement in the tree must either have just two statements immediately above it, and follow from them by Rule (F), or else be an axiom or member of $\mathscr{A}$, with nothing above it. The expression

$$
\mathscr{A} \vdash \alpha X
$$

means that there exists a deduction of $\alpha X$ from $\mathscr{A}$; and

$$
\vdash \alpha X
$$

means that there is a deduction of $\alpha X$ whose branch-tops are all axioms $\left({ }^{4}\right)$.

( $\left.{ }^{4}\right)$ We shall only be interested in deductions in which each ob in $A$ is an atom not occurring in the axiom-schemes. Throughout this paper "deduction" means this kind of deduction. 
The axiom-schemes for $\mathbf{S}$ and $\mathbf{K}$ in Definition 4 are justified in [1, p. 263], by their connection with the reduction-rules for these two combinators (see (13) later), but for the present section these reduction-rules are irrelevant. The restrictions on the unspecified axiom-schemes in Definition 4 will ensure that no ob-atom has two different principal type-schemes. Rule $(\mathrm{F})$ can be interpreted as saying that if $X$ is a function from the set $\gamma$ into the set $\delta$, and $Y$ is in $\gamma$, then $(X Y)$ is in $\delta\left({ }^{5}\right)$.

Incidentally, sometimes the ob $\mathbf{I}$ of the Introduction is included as an extra basic combinator, and sometimes it is defined in terms of $\mathbf{S}$ and $\mathbf{K}$; the present paper includes both possibilities. If $\mathbf{I}$ was included as an ob-atom, one of the unspecified axiom-schemes of Definition 4 would be (Faa)l. Some theories based on combinatory logic postulate extra axioms $\left(\boldsymbol{F}_{\alpha \beta}\right) \mathbf{I}$ for certain distinct types $\alpha$ and $\beta$ (meaning intuitively that $\alpha$ is a subset of $\beta$ ). In this case I would not have a principal type-scheme. In fact if $I$ was an atom the condition in Definition 4 that no two axiom-schemes contain the same $Y$ would not be satisfied, and if I was compound, there would be an axiom with a compound ob; thus the present paper would not apply.

From Definitions 4 and 5 it can be seen that if the obs in the statements in $\mathscr{A}$ are atoms, then the steps in a deduction

$$
\mathscr{A} \vdash \alpha X
$$

follow the construction of $X$; if $X$ is an atom, the deduction must consist of one step only, while if $X$ is $(U V)$, the deduction must be the result of joining together two deductions with the form

$$
\mathscr{A} \vdash \mathbf{F} \beta \alpha U, \quad \mathscr{A} \vdash \beta V
$$

by an application of Rule (F). Hence every ob-atom in $X$ must occur in a statement in $\mathscr{A}$ or in an axiom; and each statement in $\mathscr{A}$ which actually occurs in the deduction must have its ob-atom occurring in $X[1, \S 9 \mathrm{~B}$, Theorem 1].

EXAMPLE 1. $\vdash$ Faa(SKK).

Proof.

$$
\left\{\frac{\mathbf{F}(\mathbf{F} a(\mathbf{F}(\mathbf{F} b a) a))(\mathbf{F}(\mathbf{F} a(\mathbf{F} b a))(\mathbf{F} a a)) \mathbf{F} a(\mathbf{F}(\mathbf{F} b a) a) K}{\frac{\mathbf{F}(\mathbf{F} a(\mathbf{F} b a))(\mathbf{F} a a)(\mathbf{S K})}{\mathbf{F} a a((\mathbf{S K}) \mathbf{K})}}\right.
$$

The first two statements in this deduction, for $\mathbf{S}$ and $\mathbf{K}$, are axioms obtained by substituting F $b a$ for $b$, and $a$ for $c$, in the appropriate axiom-schemes.

$\left.{ }^{5}\right)$ If $\mathbf{S}$ and $\mathbf{K}$ are the only ob-atoms, and type-variables are prohibited from the axioms, Definition 4 is equivalent to the system in [2], and to the basic theory $\mathscr{F}_{1}(K, S)$ in $[1, \S 9 \mathrm{~A} 3]$ with restricted rule $\mathrm{F}$ and rule $\mathrm{Eq}$ omitted. 
Definition 6. Instances of deductions. For any deduction $\mathscr{Z}$, any type-schemes $\beta_{1}, \ldots, \beta_{n}$, and (distinct) type-variables $a_{1}, \ldots, a_{n}$,

$$
\left[\frac{\beta_{1}, \ldots, \beta_{n}}{a_{1}, \ldots, a_{n}}\right] \mathscr{D}
$$

is defined to be the result of simultaneously substituting $\beta_{1}, \ldots, \beta_{n}$ for $a_{1}, \ldots, a_{n}$ in all the type-schemes in $\mathscr{D}$, and it is called an instance of $\mathscr{D}$.

If the above substitution is denoted by "**", it can be seen that if $\mathscr{Q}$ is a deduction giving

$$
\left\{\alpha_{1} Y_{1}, \ldots, \alpha_{k} Y_{k}\right\} \vdash \beta X,
$$

then $\mathscr{D}^{*}$ will be a deduction giving

$$
\left\{\alpha_{1}^{*} Y_{1}, \ldots, \alpha_{k}^{*} Y_{k}\right\} \vdash \beta^{*} X .
$$

Definition 7. Principal type-schemes.

(a) Suppose each atom in $X$ occurs in an axiom-scheme; then a type-scheme $\alpha$ is a principal type-scheme (p.t.s.) of $X$ iff

(i) $\vdash \alpha X$, and

(ii) if $\vdash \beta X$, then $\beta$ is an instance of $\alpha$.

(b) If $X$ contains ob-atoms $x_{1}, \ldots, x_{n}$ (mutually distinct) which are not given types by the axiom-schemes, then $\alpha$ is said to be a p.t.s. of $X$ iff

(i) for some type-schemes $\alpha_{1}, \ldots, \alpha_{n}$,

$$
\left\{\alpha_{1} x_{1}, \ldots, \alpha_{n} x_{n}\right\} \vdash \alpha X,
$$

(ii) and if for some type-schemes $\beta_{1}, \ldots, \beta_{n}, \beta$,

$$
\left\{\beta_{1} x_{1}, \ldots, \beta_{n} x_{n}\right\} \vdash \beta X,
$$

then $\beta$ is an instance of $\alpha$.

Note. Since $\beta$ in (ii) is any type-scheme, not just a type, the notion of principal type-scheme here differs slightly from the introduction, where (ii) was only required for types $\beta$. However Theorem 1 will show that if $X$ has a type at all, $X$ must have a type-scheme which is principal in the sense of Definition 7 , and hence in the sense of the Introduction. If the number of basic types is infinite, I think the two senses are equivalent.

Definition 8. Principal deductions. Given an ob $X$, a deduction $\mathscr{D}$ of a typescheme of $X$ from a set $\mathscr{A}$ of statements is said to be a principal deduction for $X$ iff all other deductions $\mathscr{D}^{\prime}$ of type-schemes of $X$ are instances of $\mathscr{D}$ (including deductions $\mathscr{D}^{\prime}$ from other sets $\mathscr{A}^{\prime}$ of statements). The existence of a principal deduction of $\alpha X$ from a set $\mathscr{A}$ of statements is denoted by

$$
\mathscr{A} \vdash_{p} \alpha X \text {. }
$$

If $X$ has a principal deduction

$$
\left\{\alpha_{1} x_{1}, \ldots, \alpha_{n} x_{n}\right\} \vdash_{p} \alpha X
$$


(where $x_{1}, \ldots, x_{n}$ are the atoms in $X$ which do not occur in the axioms), then $\alpha$ must be a p.t.s. of $X$; because any deduction

$$
\left\{\beta_{1} x_{1}, \ldots, \beta_{n} x_{n}\right\} \vdash \beta X
$$

must be an instance of the principal deduction, and so $\beta_{1}, \ldots, \beta_{n}, \beta$. must be instances of $\alpha_{1}, \ldots, \alpha_{n}, \alpha$, respectively. (In fact, the sequence $\left\langle\beta_{1}, \ldots, \beta_{n}, \beta\right\rangle$ must be an instance of $\left\langle\alpha_{1}, \ldots, \alpha_{n}, \alpha\right\rangle$.)

Notice that for any deduction $\mathscr{D}$, the type-schemes in its statements can be laid out as a sequence, by starting at the top of the left-hand branch, working down it to just before it joins the next branch, and then going to the top of this next branch, and so on. For instance the sequence for the deduction in Example 1 is 〈type-scheme for S, first type-scheme for K, type-scheme for (SK), second typescheme for K, type-scheme for (SKK) $>$. Then a deduction is an instance of $\mathscr{D}$ if and only if its sequence is an instance of the sequence for $\mathscr{D}$ (by the same substitution).

2. Lemmas on substitution. These lemmas are stated for substitution into typeschemes, but they are also true for substitution into deductions, and into sequences of type-schemes.

Lemma 1. Any substitution

$$
\left[\frac{\beta_{1}, \ldots, \beta_{n}}{a_{1}, \ldots, a_{n}}\right] \alpha
$$

can be performed by a series of substitutions in which $n=1$, and any series of such "single" substitutions can be performed by one substitution in which $n \geqq 1$.

(See notes 7.4 and 7.6, p. 264 of [3].)

COROLlARY. Two or more successive substitutions can be performed by one substitution; hence an instance of an instance of a type-scheme $\alpha$ is an instance of $\alpha$.

Lemma 2. Any substitution for variables $a_{1}, \ldots, a_{n}$ in a type-scheme $\alpha$ can be expressed as a substitution for all the variables in $\alpha$ (by substituting themselves for the variables distinct from $a_{1}, \ldots, a_{n}$ ).

Definition 9. A trivial variant of a type-scheme $\alpha$ is any type-scheme with the form

$$
\left[\frac{b_{1}, \ldots, b_{n}}{a_{1}, \ldots, a_{n}}\right]_{\alpha}
$$

where $b_{1}, \ldots, b_{n}$ are distinct, and $a_{1}, \ldots, a_{n}$ include all the variables in $\alpha$. Trivial variants of deductions, and of sequences of type-schemes, are defined similarly.

Lemma 3. (a) The relation of being a trivial variant is reflexive, transitive and symmetric.

(b) A type-scheme $\beta$ is a trivial variant of a type-scheme $\alpha$ if and only if $\alpha$ and $\beta$ are instances of one another. 
Lemma 4. (a) If $\alpha$ is a p.t.s. of $X$, then any trivial variant of $\alpha$ is a p.t.s. of $X$, and any p.t.s. of $X$ is a trivial variant of $\alpha$.

(b) Part (a) is also true for principal deductions.

Definition 10. Highest common instance. If $\alpha$ and $\beta$ are type-schemes, and $\gamma$ is an instance of both $\alpha$ and $\beta$, such that any other instance of both $\alpha$ and $\beta$ must be an instance of $\gamma$, then $\gamma$ will be called a highest common instance (h.c.i.) of $\alpha, \beta$. Highest common instances of pairs of sequences of type-schemes are defined similarly.

Notice that if trivial variants are ignored, the h.c.i. of $\alpha, \beta$ is unique. Also, if $\alpha$ is $F \alpha_{1} \alpha_{2}$ and $\beta$ is $F \beta_{1} \beta_{2}$, then $\alpha, \beta$ have a common instance if and only if the sequences $\left\langle\alpha_{1}, \alpha_{2}\right\rangle$ and $\left\langle\beta_{1}, \beta_{2}\right\rangle$ have a common instance, and if these two sequences have a h.c.i. $\left\langle\gamma_{1}, \gamma_{2}\right\rangle$, then $\mathbf{F}_{\gamma_{1}} \gamma_{2}$ will be an h.c.i. of $\alpha, \beta$.

LEMma 5 (J. A. RoBINSON). (a) If two type-schemes $\alpha$ and $\beta$ have an instance in common, then they have a h.c.i., $\gamma$.

(b) Furthermore, given any variables $a_{1}, \ldots, a_{k}, \gamma$ can be chosen so as to contain none of $a_{1}, \ldots, a_{k}$.

(c) Parts (a) and (b) are true also for any pair $\left\langle\alpha_{1}, \ldots, \alpha_{p}\right\rangle,\left\langle\beta_{1}, \ldots, \beta_{p}\right\rangle$ of sequences of type-schemes.

Proof. Part (b) follows from (a) because if $\gamma$ were an h.c.i. of $\alpha, \beta$, any trivial variant of $\gamma$ would also be an h.c.i. of $\alpha, \beta$.

To prove (a), we may assume that $\alpha$ and $\beta$ have no variables in common, since otherwise we could find trivial variants $\alpha^{\prime}$ and $\beta^{\prime}$ with this property and use them instead of $\alpha$ and $\beta$. (By Lemma 3, they would have the same common instances as $\alpha$ and $\beta$.) Let $a_{1}, \ldots, a_{m}$ be all the variables in $\alpha$, and $b_{1}, \ldots, b_{n}$ be those in $\beta$. Then for any $\gamma_{1}, \ldots, \gamma_{m}, \delta_{1}, \ldots, \delta_{n}$, since $b_{1}, \ldots, b_{n}$ do not occur in $\alpha$, we have

Similarly,

$$
\left[\frac{\gamma_{1}, \ldots, \gamma_{m}}{a_{1}, \ldots, a_{m}}\right] \alpha \equiv\left[\frac{\gamma_{1}, \ldots, \gamma_{m}, \delta_{1}, \ldots, \delta_{n}}{a_{1}, \ldots, a_{m}, b_{1}, \ldots, b_{n}}\right] \alpha \text {. }
$$

$$
\left[\frac{\delta_{1}, \ldots, \delta_{n}}{b_{1}, \ldots, b_{n}}\right] \beta \equiv\left[\frac{\gamma_{1}, \ldots, \gamma_{m}, \delta_{1}, \ldots, \delta_{n}}{a_{1}, \ldots, a_{m}, b_{1}, \ldots, b_{n}}\right] \beta
$$

thus we can look at any common instance of $\alpha, \beta$ as having been obtained by performing the same substitution on $\alpha$ as on $\beta$. Now J. A. Robinson in [4, p. 32] (Unification Theorem) has given an algorithm which allows us to decide whether in the above circumstances, $\alpha$ and $\beta$ have an instance in common, and if they do, to construct a h.c.i. for them. This proves (a).

To prove (c), notice first that if two sequences $\left\langle\alpha_{1}, \ldots, \alpha_{p}\right\rangle$ and $\left\langle\beta_{1}, \ldots, \beta_{q}\right\rangle$ have an instance in common, then they must have the same number of terms. Let $\theta$ be a basic type, and define

$$
\begin{aligned}
\alpha & \equiv F_{\alpha_{1}}\left(F_{\alpha_{2}}\left(\cdots\left(F\left(\boldsymbol{F}_{p} \theta\right) \cdots\right)\right)\right. \\
\beta & \equiv \mathbf{F} \beta_{1}\left(\mathbf{F} \beta_{2}\left(\cdots\left(F \beta_{p} \theta\right) \cdots\right)\right) .
\end{aligned}
$$


Then $\alpha$ and $\beta$ have a common instance if and only if the given sequences have a common instance (since nothing can be substituted for $\theta$ ), and if the h.c.i. of $\alpha$ and $\beta$ is

$$
\mathbf{F}_{\gamma_{1}}\left(\mathbf{F}_{\gamma_{2}}\left(\cdots\left(\mathbf{F}_{\gamma_{p}} \theta\right) \cdots\right)\right)
$$

then the h.c.i. of the given sequences will be

$$
\left\langle\gamma_{1}, \ldots, \gamma_{p}\right\rangle \text {. }
$$

LEMMA 6. Given a sequence $\left\langle\phi_{1}, \ldots, \phi_{m}, \psi_{1}, \ldots, \psi_{n}\right\rangle$ of type-schemes, let $a_{1}, \ldots, a_{p}$ be all the variables in $\left\langle\phi_{1}, \ldots, \phi_{m}\right\rangle$ and $b_{1}, \ldots, b_{q}$ be the variables (if any) that are in $\left\langle\psi_{1}, \ldots, \psi_{n}\right\rangle$ but not in $\left\langle\phi_{1}, \ldots, \phi_{m}\right\rangle$; let " *" denote the effect of substituting for $a_{1}, \ldots, a_{p}$ certain type-schemes containing none of $b_{1}, \ldots, b_{q}$. Then if $\left\langle\lambda_{1}, \ldots, \lambda_{m}\right.$, $\left.\mu_{1}, \ldots, \mu_{n}\right\rangle$ is an instance of the given sequence, and $\left\langle\lambda_{1}, \ldots, \lambda_{m}\right\rangle$ is an instance of $\left\langle\phi_{1}^{*}, \ldots, \phi_{m}^{*}\right\rangle$, the whole sequence $\left\langle\lambda_{1}, \ldots, \lambda_{m}, \mu_{1}, \ldots, \mu_{n}\right\rangle$ will be an instance of $\left\langle\phi_{1}^{*}, \ldots, \phi_{m}^{*}, \psi_{1}^{*}, \ldots, \psi_{n}^{*}\right\rangle$.

Proof. For ease of reading, suppose $m=n=1$. Let $\alpha_{1}, \ldots, \alpha_{p}$ be the type-schemes substituted for $a_{1}, \ldots, a_{p}$ respectively by the substitution "*"; let $e_{1}, \ldots, e_{r}$ be all the variables in these type-schemes. Now for some $\gamma_{1}, \ldots, \gamma_{p}, \delta_{1}, \ldots, \delta_{q}$,

$$
\left\langle\lambda_{1}, \mu_{1}\right\rangle \equiv\left[\frac{\gamma_{1}, \ldots, \gamma_{p}, \delta_{1}, \ldots, \delta_{q}}{a_{1}, \ldots, a_{p}, b_{1}, \ldots, b_{q}}\right]\left\langle\phi_{1}, \psi_{1}\right\rangle .
$$

But also, for some $\varepsilon_{1}, \ldots \varepsilon_{r}$,

$$
\begin{aligned}
\lambda_{1} & \equiv\left[\frac{\varepsilon_{1}, \ldots, \varepsilon_{r}}{e_{1}, \ldots, e_{r}}\right] \phi_{1}^{*} \\
& \equiv\left[\frac{\varepsilon_{1}, \ldots, \varepsilon_{r}}{e_{1}, \ldots, e_{r}}\right]\left[\frac{\alpha_{1}, \ldots, \alpha_{p}}{a_{1}, \ldots, a_{p}}\right] \phi_{1} \\
& \equiv\left[\frac{\alpha_{1}^{\prime}, \ldots, \alpha_{p}^{\prime}}{a_{1}, \ldots, a_{p}}\right] \phi_{1},
\end{aligned}
$$

where for each $i$, $\alpha_{i}^{\prime}$ is the result of substituting $\varepsilon_{1}, \ldots, \varepsilon_{r}$ for $e_{1}, \ldots, e_{r}$ respectively in $\alpha_{i}$. Therefore by the previous expression for $\left\langle\lambda_{1}, \mu_{1}\right\rangle$,

Hence

$$
\gamma_{i} \equiv \alpha_{i}^{\prime}
$$

$$
\begin{aligned}
\left\langle\lambda_{1}, \mu_{1}\right\rangle & \equiv\left[\frac{\alpha_{1}^{\prime}, \ldots, \alpha_{p}^{\prime}, \delta_{1}, \ldots, \delta_{q}}{a_{1}, \ldots, a_{p}, b_{1}, \ldots, b_{q}}\right]\left\langle\phi_{1}, \psi_{1}\right\rangle \\
& \equiv\left[\frac{\varepsilon_{1}, \ldots, \varepsilon_{r}, \delta_{1}, \ldots, \delta_{q}}{e_{1}, \ldots, e_{r}, b_{1}, \ldots, b_{q}}\right]\left[\frac{\alpha_{1}, \ldots, \alpha_{p}, b_{1}, \ldots, b_{q}}{a_{1}, \ldots, a_{p}, b_{1}, \ldots, b_{q}}\right]\left\langle\phi_{1}, \psi_{1}\right\rangle
\end{aligned}
$$

since $e_{1}, \ldots, e_{r}, b_{1}, \ldots, b_{q}$ are distinct, and $\alpha_{1}, \ldots, \alpha_{p}$ do not contain $b_{1}, \ldots, b_{q}$. Thus $\left\langle\lambda_{1}, \mu_{1}\right\rangle$ is an instance of $\left\langle\phi_{1}^{*}, \psi_{1}^{*}\right\rangle$. 
3. Existence of principal type-schemes. In future, whenever a list (e.g. " $x_{1}, \ldots, x_{n}$ ") of ob-atoms is mentioned, the atoms will be assumed to be distinct.

THEOREM 1. Let $X$ be an $o b$, and $x_{1}, \ldots, x_{t}(t \geqq 0)$ be the ob-atoms in $X$ that are not given types by the axiom-schemes; if a type-scheme for $X$ can be deduced from some assumptions, then there exists a principal deduction $\mathscr{Z}$ for $X$ (and hence $X$ has a p.t.s.).

Proof. We use induction on the construction of $X$.

If $X$ is an atom occurring in an axiom-scheme $\alpha X$, choose $\mathscr{D}$ to be the one-step deduction

$$
\alpha X
$$

This is principal because every other deduction must have the form $\beta X$, where $\beta$ is an instance of $\alpha$.

If $X$ is an atom not given a type by an axiom-scheme, choose $\mathscr{D}$ to be the one-step deduction

$$
a X \text {, }
$$

where $a$ is any type-variable. This is obviously principal. Thus the p.t.s. of $X$ is a single variable in this case.

Now suppose $X$ is $(U V)$; let $u_{1}, \ldots, u_{p}$ be those of $x_{1}, \ldots, x_{t}$ which occur in $U$ but not in $V, w_{1}, \ldots, w_{r}$ be those which occur in both $U$ and $V, v_{1}, \ldots, v_{q}$ be those which occur in $V$ but not in $U$. Let the given deduction of a type-scheme for $X$ have the form

$$
\left\{\lambda_{1} u_{1}, \ldots, \lambda_{p} u_{p}, \mu_{1} v_{1}, \ldots, \mu_{q} v_{q}, \nu_{1} w_{1}, \ldots, \nu_{r} w_{r}\right\} \vdash \beta X .
$$

This deduction must be the result of applying Rule (F) to two deductions with the forms

$$
\begin{aligned}
& \left\{\lambda_{1} u_{1}, \ldots, \lambda_{p} u_{p}, \nu_{1} w_{1}, \ldots, v_{r} w_{r}\right\} \vdash \mathbf{F} \alpha \beta U, \\
& \left\{\mu_{1} v_{1}, \ldots, \mu_{q} v_{q}, \nu_{1} w_{1}, \ldots, \nu_{r} w_{r}\right\} \vdash \alpha V .
\end{aligned}
$$

By the induction hypothesis, $U$ and $V$ have principal deductions $\mathscr{D}_{1}$ and $\mathscr{D}_{2}$; suppose these have the forms

$$
\begin{gathered}
\left\{\theta_{1} u_{1}, \ldots, \theta_{p} u_{p}, \psi_{1} w_{1}, \ldots, \psi_{r} w_{r}\right\} \vdash_{p} \pi U, \\
\left\{\phi_{1} v_{1}, \ldots, \phi_{q} v_{q}, \chi_{1} w_{1}, \ldots, \chi_{r} w_{r}\right\} \vdash_{p} \zeta V .
\end{gathered}
$$

Let $a_{1}, \ldots, a_{k}$ be the type-variables in $\mathscr{D}_{1}$ that do not occur in $\left\langle\psi_{1}, \ldots, \psi_{r}, \pi\right\rangle$,

$b_{1}, \ldots, b_{l}$ be all the type-variables in $\left\langle\psi_{1}, \ldots, \psi_{r}, \pi\right\rangle$,

$c_{1}, \ldots, c_{m}$ be all the type-variables in $\left\langle\chi_{1}, \ldots, \chi_{r}, \zeta\right\rangle$,

$d_{1}, \ldots, d_{n}$ be the type-variables in $\mathscr{Q}_{2}$ that are not in $\left\langle\chi_{1}, \ldots, \chi_{r}, \zeta\right\rangle$. Let $g$ be a new type-variable, distinct from all those above.

The principal deduction for $(U V)$ is constructed as follows. First choose $\mathscr{D}_{1}$ and $\mathscr{D}_{2}$ so that all the above variables are distinct (this is possible by Lemma $4(\mathrm{~b})$ ). 
Then find an h.c.i. of the two sequences

$$
\left\langle\psi_{1}, \ldots, \psi_{r}, \pi\right\rangle, \quad\left\langle\chi_{1}, \ldots, \chi_{r},(\mathbf{F} \zeta g)\right\rangle
$$

which contains none of $a_{1}, \ldots, a_{k}, d_{1}, \ldots, d_{n}$ (this is possible by Lemma $5(\mathrm{c}$ ), since the two sequences have a common instance $\left\langle\nu_{1}, \ldots, \nu_{r},\left(F_{\alpha \beta}\right\rangle\right)$ by (2) and (3) above). Suppose this h.c.i. is

$$
\left\langle\omega_{1}, \ldots, \omega_{r},(\mathbf{F} \sigma \tau)\right\rangle, \equiv\left\langle\psi_{1}^{*}, \ldots, \psi_{r}^{*}, \pi^{*}\right\rangle ; \equiv\left\langle\chi_{1}, \ldots, \chi_{r}^{\circ},\left(\mathbf{F} \zeta^{\circ} \tau\right)\right\rangle
$$

where "*" denotes the substitution of certain type-schemes for $b_{1}, \ldots, b_{1}$ only, and "०" for $c_{1}, \ldots, c_{m}$ only. Then construct the two deductions $\mathscr{D}_{1}^{*}$ and $\mathscr{D}_{2}^{\circ}$, which give

$$
\begin{gathered}
\left\{\theta_{1}^{*} u_{1}, \ldots, \theta_{p}^{*} u_{p}, \psi_{1}^{*} w_{1}, \ldots, \psi_{r}^{*} w_{r}\right\} \vdash \mathbf{F}_{\sigma \tau} U, \\
\left\{\phi_{1}^{\circ} v_{1}, \ldots, \phi_{q}^{\circ} v_{q}, \chi_{1}^{\circ} w_{1}, \ldots, \chi_{r}^{\circ} w_{r}\right\} \vdash \zeta^{\circ} V .
\end{gathered}
$$

Since $\sigma \equiv \zeta^{\circ}$ and $\psi_{i}^{*} \equiv \chi_{i}^{\circ}$ for each $i$, these two deductions can be combined by Rule (F) to form one deduction, giving

$$
\left\{\theta_{1}^{*} u_{1}, \ldots, \theta_{p}^{*} u_{p}, \psi_{1}^{*} w_{1}, \ldots, \psi_{r}^{*} w_{r}, \phi_{1}^{\circ} v_{1}, \ldots, \phi_{q}^{\circ} v_{q}\right\} \vdash \tau(U V) .
$$

We shall see that this is a principal deduction for $(U V)$; to do this, we must prove that any deduction with the form (1) is an instance of $(9)\left({ }^{6}\right)$. For this it is enough to show that the deduction (2) is an instance of (7), and (3) is an instance of (8), both by the same substitution. Notice that since (4) and (5) are principal, (2) and (3) are instances of (4) and (5) respectively.

Now let $\mathscr{S}_{2}, \mathscr{S}_{3}, \mathscr{S}_{4}, \mathscr{S}_{5}$ be the sequences for the deductions (2), (3), (4), (5) respectively (see the end of $\S 1$ ). To show that (2), (3) are instances of (7), (8) both by the same substitution, it is enough to show that the sequence $\left\langle\mathscr{S}_{2}, \mathscr{S}_{3}\right\rangle$ (formed by putting the members of $\mathscr{S}_{3}$ after the members of $\mathscr{S}_{2}$ ) is an instance of the sequence $\left\langle\mathscr{S}_{4}{ }^{*}, \mathscr{S}_{5}{ }^{\circ}\right\rangle$, since $\mathscr{S}_{4}{ }^{*}$ is the sequence for (7), and $\mathscr{S}_{5}{ }^{\circ}$ corresponds to (8). We shall use Lemma 6.

Since no variables are common to (4) and (5), and (2), (3) are instances of (4), (5), the sequence $\left\langle\mathscr{S}_{2}, \mathscr{S}_{3}\right\rangle$ is an instance of $\left\langle\mathscr{S}_{4}, \mathscr{S}_{5}\right\rangle$. Hence the sequence

is an instance of

$$
\left\langle\mathscr{S}_{2}, \mathscr{S}_{3}, \nu_{1}, \ldots, \nu_{r},(\mathbf{F} \alpha \beta), \nu_{1}, \ldots, \nu_{r},(\mathbf{F} \alpha g)\right\rangle
$$

$$
\left\langle\mathscr{S}_{4}, \mathscr{S}_{5}, \psi_{1}, \ldots, \psi_{r}, \pi, \chi_{1}, \ldots, \chi_{r},(\mathbf{F} \zeta g)\right\rangle
$$

because $\nu_{1}, \ldots, \nu_{r},\left(\boldsymbol{F}_{\alpha \beta} \beta\right)$ occur in $\mathscr{S}_{2}$ at positions corresponding to $\psi_{1}, \ldots, \psi_{r}, \pi$ in $\mathscr{S}_{4}$, and similarly for the other members. Also, by (6),

$\left({ }^{6}\right)$ Here the relevant deductions will be called "(1)," "(9)," etc., though strictly speaking (1) and (9) are not deductions but only the hypotheses and conclusions of deductions. Thus for instance, type-variables may occur in the deduction giving (1) but not appear in (1) itself (if they occurred in axioms used in the deduction); in Example 1, $b$ is such a variable. 


$$
\begin{aligned}
\left\langle\omega_{1}, \ldots, \omega_{r},(\mathbf{F} \sigma \tau), \omega_{1}, \ldots, \omega_{r},(\mathbf{F} \sigma \tau)\right\rangle & \equiv\left\langle\psi_{1}^{*}, \ldots, \psi_{r}^{*}, \pi^{*}, \chi_{1}^{\circ}, \ldots, \chi_{r}^{\circ},\left(\mathbf{F} \zeta^{\circ} \tau\right)\right\rangle \\
& \equiv\left\langle\psi_{1}, \ldots, \psi_{r}, \pi, \chi_{1}, \ldots, \chi_{r},(\mathbf{F} \zeta g)\right\rangle^{+}
\end{aligned}
$$

where " +" denotes the result of simultaneously performing substitution * on $b_{1}, \ldots, b_{1}$, and ${ }^{\circ}$ on $c_{1}, \ldots, c_{m}$, and substituting $\tau$ for $g$. By the definition of (6), if there are any type-variables in $\mathscr{S}_{4}$ or $\mathscr{S}_{5}$ which do not occur in

$$
\left\langle\psi_{1}, \ldots, \psi_{r}, \pi, \chi_{1}, \ldots, \chi_{r},(\mathbf{F} \zeta g)\right\rangle,
$$

they do not occur in the above sequence. Hence by Lemma 6,

$$
\left\langle\mathscr{S}_{2}, \mathscr{S}_{3}, \nu_{1}, \ldots, v_{r},(\mathbf{F} \alpha \beta), \nu_{1}, \ldots, v_{r},(\mathbf{F} \alpha g)\right\rangle
$$

is an instance of

$$
\left\langle\mathscr{S}_{4}^{+}, \mathscr{S}_{5}^{+}, \psi_{1}^{+}, \ldots, \psi_{r}^{+}, \pi^{+}, \chi_{1}^{+}, \ldots, \chi_{r}^{+},(\mathbf{F} \zeta g)^{+}\right\rangle .
$$

Hence $\left\langle\mathscr{S}_{2}, \mathscr{S}_{3}\right\rangle$ is an instance of $\left\langle\mathscr{S}_{4}^{+}, \mathscr{S}_{5}^{+}\right\rangle$; since this is the same as $\left\langle\mathscr{S}_{4}^{*}, \mathscr{S}_{5}^{\circ}\right\rangle$ the proof is complete.

REMARK. In this proof the p.t.s. $\pi$ of $U$ in (4) may be either compound or a variable. (It cannot be a basic type since it has an instance $\boldsymbol{F}_{\alpha \beta} \beta$ which is compound.) The variable $g$ was introduced just to treat these two cases simultaneously, and when $\pi$ is compound, $g$ is not really necessary.

COROLlaRY 1.1. There is an effective way of deciding for each $X$ whether or not $X$ has a type-scheme, and if it has one, for calculating its p.t.s., and its principal deduction. (Assuming that atoms can be effectively distinguished from each other and from compound obs, and that substitutions can be effectively performed, etc.)

Proof. If $X$ is an atom, then the p.t.s. of $X$ either is given by axiom-scheme, or else is a single type-variable.

Suppose $X$ is $(U V)$; if $U$ or $V$ has no type-schemes, then $X$ has none. If $U$ and $V$ have principal deductions with the forms (4) and (5), then Robinson's theorem (Lemma 5) gives an effective test of whether the h.c.i. (6) is constructible; if it is, the principal deduction for $X$ is (9) and the p.t.s. is $\tau$ therein; if not, $X$ has no type-schemes at all.

COROLlaRY 1.2. For every p.t.s. $\alpha$ of $X$, there exists a principal deduction of $\alpha X$.

Proof. By Theorem 1, $X$ has a principal deduction; let $\beta X$ be the statement it deduces. By Lemma 4(a), $\beta$ is a trivial variant of $\alpha$, and hence suitable changes of the variables in the deduction of $\beta X$ will form a principal deduction of $\alpha X$.

The following two corollaries show how to calculate the p.t.s. of $(U V)$ from those of $U$ and $V$, in the important case that all atoms common to $U$ and $V$ are given types by the axiom-schemes. In this ase $r=0$ in the proof of the theorem, and so the principal deduction is constructed by finding an h.c.i. of $\pi$ and $\mathbf{F} \zeta g$, and combining the corresponding deductions (7) and (8). 
COROLlary 1.3. Suppose that all the atoms common to $U$ and $V$ are given types by the axiom-schemes; if

$$
\left\{\theta_{1} u_{1}, \ldots, \theta_{p} u_{p}\right\} \vdash_{p} \mathbf{F} \xi \eta U
$$

and

$$
\left\{\phi_{1} v_{1}, \ldots, \phi_{q} v_{q}\right\} \vdash_{p} \zeta V
$$

and $\xi, \zeta$ have an h.c.i. $\xi^{*} \equiv \zeta^{\circ}$ whose new variables (i.e. those not in $\xi$ ) do not occur in $\eta$, then the result $\eta^{*}$, of making exactly the same substitutions in $\eta$ as changed $\xi$ to $\xi^{*}$, is a p.t.s. of $(U V)$.

Proof. By Lemma $4 \mathrm{~b}$ we may replace the deduction ( $5 \mathrm{a}$ ) by one with no variables in common with (4a). Suppose this deduction gives

$$
\left\{\phi_{1}^{\prime} v_{1}, \ldots, \phi_{q}^{\prime} v_{q}\right\} \vdash_{p} \zeta^{\prime} V .
$$

The type-scheme $\xi^{*}$ will be an h.c.i. of $\xi$ and $\zeta^{\prime}$.

Now $\mathbf{F} \xi^{*} \eta^{*}$ is an h.c.i. of $\mathbf{F} \xi \eta$ and $\mathbf{F} \zeta^{\prime} g$, if $g$ does not occur in $\zeta^{\prime}$. It is certainly a common instance. Also if $\boldsymbol{F}_{\alpha \beta} \beta$ is any other common instance, $\alpha$ must be an instance of $\xi^{*}$ since $\xi^{*}$ is an h.c.i. of $\xi$ and $\zeta^{\prime}$; hence by Lemma 6, F $\alpha \beta$ is an instance of $\mathbf{F} \xi^{*} \eta^{*}$. (Lemma 6 is here applied to the pairs $\langle\xi, \eta\rangle,\left\langle\xi^{*}, \eta^{*}\right\rangle$ and $\langle\alpha, \beta\rangle$.)

To follow the construction in the proof of Theorem 1, with $r=0$ and $\pi \equiv \mathbf{F} \xi \eta$ in the present case, the first step is to find an h.c.i. of $\mathbf{F} \xi \eta$ and $\mathbf{F} \boldsymbol{\zeta}^{\prime} \boldsymbol{g}$ that does not contain $a_{1}, \ldots, a_{k}, d_{1}, \ldots, d_{n}$ (see Theorem 1). If $\mathbf{F} \xi^{*} \eta^{*}$ is not suitable, we can find a suitable trivial variant $\boldsymbol{F} \xi^{\prime \prime} \eta^{\prime \prime}$ of it. Then the principal deduction (9) for (UV) is formed by combining certain instances of (4a) and (5a)', and shows that $\eta^{\prime \prime}$ is a p.t.s. of $U V$. Hence $\eta^{*}$ is a p.t.s., since it is a trivial variant of $\eta^{\prime \prime}$.

COROLlary 1.4. If in Corollary 1.3 all the variables in the hypotheses of (4a) occur in $\mathbf{F} \xi \eta$, and all those in the hypotheses of (5a) occur in $\zeta$, then

$$
\left\{\theta_{1}^{*} u_{1}, \ldots, \theta_{p}^{*} u_{p}, \phi_{1}^{\circ} v_{1}, \ldots, \phi_{q}^{\circ} v_{q}\right\} \vdash_{p} \eta^{*}(U V) .
$$

Proof. In the proof of Corollary $1.3, a_{1}, \ldots, a_{k}$ are the variables in the deduction for $U$ which do not occur in $\mathbf{F} \xi_{\eta}$; hence by the assumption they do not occur in any of $\theta_{1}, \ldots, \theta_{p}, \mathbf{F} \xi \eta$. Therefore by Lemma $4 \mathrm{~b}$ a principal deduction can be found which still gives (4a) but with $a_{1}, \ldots, a_{k}$ not occurring in $\mathbf{F} \xi^{*} \eta^{*}$. Similarly we may assume that $d_{1}, \ldots, d_{n}$ in $(5 a)^{\prime}$ do not occur in $\mathbf{F} \xi^{*} \eta^{*}$. Therefore $\mathbf{F} \xi^{*} \eta^{*}$ is a suitable h.c.i. for the proof of Corollary 1.3. To construct the principal deduction (9) for $U V$, we first perform in (5a) the substitution which changes $\zeta^{\prime}$ to $\zeta^{\circ}$; since all the variables in each $\phi_{i}^{\prime}$ occur in $\zeta^{\prime}$, this changes $\phi_{i}^{\prime}$ to $\phi_{i}^{\circ}$, giving a deduction

$$
\left\{\phi_{1}^{\circ} v_{1}, \ldots, \phi_{q}^{\circ} v_{q}\right\} \vdash \zeta^{\circ} V .
$$

Next we perform substitution "*" in (4a) and combine the result with the above deduction by Rule (F), completing the corollary. 
Corollary 1.5. If in (4) and (5) of Theorem $1, \pi$ has the form $\mathbf{F} \xi \eta$ and $\zeta \equiv \xi$, $\psi_{i} \equiv \chi_{i}$ for all $i$ (these being the only exceptions to the assumption that (4) and (5) have no variables in common), then a principal deduction for UV is obtained by simply applying Rule (F) to (4) and (5).

Proof. To construct a principal deduction as in Theorem 1, first change all the variables in (5) that occur in $\zeta, \chi_{1}, \ldots, \chi_{r}$ to distinct new ones not occurring in (4). This leaves (4) and (5) with no variables in common. Then in (6), take "*" to be the identity substitution and " $\circ$ " to be the reverse of the above change of variables, and $\tau \equiv \eta$. Then (7) and (8) are the given (4) and (5); hence the result.

EXAMPLE 2. Let $x, y, z$ be distinct ob-atoms not given types by the axiomschemes; then

$$
\{\mathbf{F} a b x, a y, a z\} \vdash_{p} b(\mathbf{K}(x y)(x z)) .
$$

Proof. Let $a, b, c, d, e, g_{1}, g_{2}$ be distinct type-variables. Principal deductions for $x$ and $y$ are

$$
c x, \quad a y \text {. }
$$

To get a principal deduction for $(x y)$ by the construction in Theorem 1, notice that an h.c.i. of $c$ and $\left(F a g_{1}\right)$ is just (Fag $)$ itself; hence, replacing $c$ by $\left(F a g_{1}\right)$ and combining the two deductions by Rule (F) gives a principal deduction

$$
\frac{\mathbf{F} a g_{1} x \quad a y}{g_{1}(x y)} \text {. }
$$

A principal deduction for $\mathbf{K}$ is the one step $\mathbf{F} g_{1}\left(F b g_{1}\right) \mathbf{K}$; combining this with (10) by Corollary 1.5 gives the principal deduction

$$
\frac{F g_{1}\left(F b g_{1}\right) K \frac{F a g_{1} x \quad a y}{g_{1}(x y)}}{F b g_{1}(K(x y))}
$$

Now just as for $(x y)$, a principal deduction for $(x z)$ is

$$
\frac{\text { Fed } x \quad e z}{d(x z)}
$$

To obtain the result, combine (11) and (12). No type-variables are common to both, and the only ob-atom they have in common is $x$; hence we must find an h.c.i. of the sequences

$$
\left\langle\left(\mathbf{F} a g_{1}\right),\left(\mathbf{F} b g_{1}\right)\right\rangle, \quad\left\langle(\mathbf{F} c d),\left(\mathbf{F} d g_{2}\right)\right\rangle .
$$

All variables in (10) and (11) occur in these sequences, so there is no restriction on the h.c.i. we can use. A suitable one is

$$
\langle(\mathbf{F} a b),(\mathbf{F} b b)\rangle,
$$


obtained by substituting $b$ for $g_{1}$ in the former sequence, and $a, b, b$ for $c, d, g_{2}$ in the latter sequence. Making the same substitutions in (11) and (12) and combining by Rule (F) gives the principal deduction

$$
\{\mathbf{F} a b x, a y, a z\} \vdash_{p} b(\mathbf{K}(x y)(x z)) .
$$

EXAmple 3. If I $\equiv \mathbf{S K K}$, then $\vdash_{p}$ Faal.

Proof. By the axiom-schemes,

$$
\vdash_{p} \mathbf{F}(\mathbf{F} a(\mathbf{F} b c))(\mathbf{F}(\mathbf{F} a b)(\mathbf{F} a c)) \mathbf{S}, \quad \vdash_{p} \mathbf{F} a(\mathbf{F} b a) \mathbf{K} .
$$

By Corollary 1.3, since $\mathbf{F} a(\mathbf{F} b a)$ is an h.c.i. of $\mathbf{F} a(\mathbf{F} b c)$ and $\mathbf{F} a(\mathbf{F} b a)$, substituting $a$ for $c$ in the p.t.s. of $\mathbf{S}$ and applying Rule $(\mathrm{F})$ gives

$$
\vdash_{p} \mathbf{F}(\mathbf{F} a b)(\mathbf{F} a a)(\mathbf{S K}) .
$$

Now $\mathbf{F} a(\mathbf{F} b a)$ is an h.c.i. of $\mathbf{F} a b$ and $\mathbf{F} a(\mathbf{F} b a)$, so by Corollary 1.3 again (substituting Fba for $b$ in the p.t.s. of SK),

$$
\vdash_{p} \text { Faa(SKK). }
$$

But we can say more than this: in the first application of Corollary 1.3 above, there are no $a_{1}, \ldots, a_{k}, d_{1}, \ldots, d_{n}$ to worry about in the proof of the corollary, and similarly for the second application (since every variable in the deduction for SK occurs in its conclusion), so the above proof actually gives us a principal deduction for SKK. This deduction is the same as in Example 1.

EXAmple 4. The ob SII has no type-schemes at all.

Proof. By Example 3,

$$
\vdash_{p} \mathbf{F}(\mathbf{F} a(\mathbf{F} b c))((\mathbf{F} a b)(\mathbf{F} a c)) \mathbf{S}, \quad \vdash_{p} \mathbf{F} a a \mathbf{l} .
$$

Now $\mathbf{F}(\mathbf{F} b c)(\mathbf{F} b c)$ is an h.c.i. of $\mathbf{F} a(\mathbf{F} b c)$ and $\mathbf{F} a a$, so by Corollary 1.3 and substituting $\mathbf{F} b c$ for $a$,

$$
\vdash_{p} \mathbf{F}(\mathbf{F}(\mathbf{F} b c) b)(\mathbf{F}(\mathbf{F} b c) c)(\mathbf{S I})
$$

If SII had any type-schemes, the construction in Theorem 1 would give us a p.t.s. But this construction involves finding an h.c.i. of

$$
\mathbf{F}(\mathbf{F}(\mathbf{F} b c) b)(\mathbf{F}(\mathbf{F} b c) c), \quad \mathbf{F}(\mathbf{F} a a) g,
$$

and the existence of such an h.c.i. would imply that

$$
\mathbf{F}(\mathbf{F} b c) b, \quad \mathbf{F} a a
$$

have instances in common, which is impossible. Thus the construction fails, so SII has no type-schemes.

4. The principal type-scheme of $[x] . M$. The main result of this section is a lemma for use in the proof of Theorem 3, $\$ 6$. From now on the ob-atoms will be assumed to include $\mathbf{S}$ and $\mathbf{K}$, together with some $o b$-variables, which are not given 
types by the axiom-schemes. (These variables can be adjoined as extra ob-atoms if they are not already present.) Letters " $u$ ", " $v$ ", " $w$ ", " $x$ ", " $y$ ", " $z$ " will denote these variables, and for ease of reading it will be assumed that all the ob-atoms which do not occur in the axiom-schemes are variables.

Definition 11. Reduction and equality. An ob $X$ is said to reduce to $Y$ (" $X \geqq Y$ ") iff either $Y \equiv X$ or else $Y$ is the result of applying to $X$ a series of replacements with the forms (for any $U, V, W$ ):

$\mathbf{S} U V W$ may be replaced by $U W(V W)$,

$\mathbf{K} U V$ may be replaced by $U$.

The properties and purpose of reduction are explained in [1]. The phrase " $X$ is equal to $Y$ " (" $X=Y$ ") is defined to mean that $Y$ is the result of applying to $X$ a series of replacements as above, and reversed replacements.

It is proved in [1, $\$ 9 \mathrm{C} 2$, Theorem 2] that

$$
\text { if } X \geqq Y \text { and }\left\{\alpha_{1} x_{1}, \ldots, \alpha_{n} x_{n}\right\} \vdash \beta X \text {, then }\left\{\alpha_{1} x_{1}, \ldots, \alpha_{n} x_{n}\right\} \vdash \beta Y \text {. }
$$

Definition 12. [x].M. For each ob-variable $x$ and each ob $M$, an ob called " $[x] . M$ " can be defined as follows, by induction on the construction of $M$.

(i) $[x] . x \equiv \mathbf{I}$, that is, SKK;

(ii) $[x] . M \equiv \mathbf{K} M$ if $M$ does not contain $x$;

(iii) $[x] .(U V) \equiv((\mathbf{S}[x] . U)[x] . V)$ if $(U V)$ contains $x$.

This definition has the property that for all $x, M, N$,

$$
([x] . M) N \geqq[N / x] M,
$$

where $[N / x] M$ is just the result of substituting $N$ for every occurrence of $x$ in $M$. Thus $[x] . M$ represents the function of $x$ defined by $M$; see $[1, \S 6 A]$ for details. Since $[x] . M$ represents a function of $x$, we should expect to be able to prove that if $M$ has type $\eta$, deduced by giving $x$ a type $\xi$, then $[x] . M$ has type $\mathbf{F} \xi \eta$. More precisely, letting $y_{1}, \ldots, y_{n}$ be the other ob-variables in $M$,

$$
\begin{gathered}
\text { if for some types } \alpha_{1}, \ldots, \alpha_{n}, \xi, \eta,\left\{\alpha_{1} y_{1}, \ldots, \alpha_{n} y_{n}, \xi x\right\} \vdash \eta M, \\
\text { then }\left\{\alpha_{1} y_{1}, \ldots, \alpha_{n} y_{n}\right\} \vdash \mathbf{F} \xi \eta([x], M) .
\end{gathered}
$$

This is actually proved in $[1, \S 9 \mathrm{D}$, Corollary 1.1$]$; the theorem below is its analogue for principal deductions.

THEOREM 2. (a) Suppose that $x, y_{1}, \ldots, y_{n}$ are the only ob-variables in $M$. If

then

$$
\left\{\alpha_{1} y_{1}, \ldots, \alpha_{n} y_{n}, \xi x\right\} \vdash_{p} \eta M,
$$

$$
\left\{\alpha_{1} y_{1}, \ldots, \alpha_{n} y_{n}\right\} \vdash_{p} \mathbf{F} \xi \eta([x] . M) .
$$

(b) Suppose that $M$ contains only $y_{1}, \ldots, y_{n}$ and not $x$; if

$$
\left\{\alpha_{1} y_{1}, \ldots, \alpha_{n} y_{n}\right\} \vdash_{p} \eta M,
$$

then

$$
\left\{\alpha_{1} y_{1}, \ldots, \alpha_{n} y_{n}\right\} \vdash_{p} F_{e \eta}([x] . M)
$$

where $e$ is any type-variable not occurring in $\eta$. 
Proof. For part (b): $[x] . M$ is $\mathbf{K} M$, and

$$
\vdash_{p} \mathbf{F} a(\mathbf{F} e a) K
$$

Therefore by the given deduction and Corollary 1.3, since $\eta$ is an h.c.i. of $\eta$ and $a$,

$$
\left\{\alpha_{1} y_{1}, \ldots, \alpha_{n} y_{n}\right\} \vdash_{p} \mathbf{F} e \eta(\mathbf{K} M) .
$$

For part (a) we use Curry's proof (see an earlier comment). Firstly, since $M$ has a type-scheme it must have a type, since all the type-variables in a deduction can be replaced by basic types. Therefore by (15), $[x] . M$ has a type. Every type of $[x] . M$ must be compound; this is true if $M$ is $x$, since then $[x] . M$ is $I$; when $M$ has the form $U V$, then

$$
[x] . M \equiv(\mathbf{S}[x] . U)[x] . V
$$

and by the axiom-scheme for $\mathbf{S}$, the p.t.s. of this ob (if it has one) must be an instance of $(F a c)$, which is compound. Hence the p.t.s. of $[x] . M$ is compound, so the principal deduction for $[x], M$ must have the form

$$
\left\{\beta_{1} y_{1}, \ldots, \beta_{n} y_{n}\right\} \vdash_{p} \mathbf{F} \gamma \delta([x] . M) .
$$

By (15) and the given deduction for $M$ we have a deduction

$$
\left\{\alpha_{1} y_{1}, \ldots, \alpha_{n} y_{n}\right\} \vdash \mathbf{F} \xi \eta([x] . M) .
$$

This must be an instance of (16), since (16) is principal; hence

$$
\left\langle\alpha_{1}, \ldots, \alpha_{n},(F \xi \eta)\right\rangle \text { is an instance of }\left\langle\beta_{1}, \ldots, \beta_{n},(\mathbf{F} \gamma \delta)\right\rangle \text {. }
$$

Now by (16) and Rule (F),

$$
\left\{\beta_{1} y_{1}, \ldots, \beta_{n} y_{n}, \gamma x\right\} \vdash \delta(([x] . M) x),
$$

and so by (14) and (13),

$$
\left\{\beta_{1} y_{1}, \ldots, \beta_{n} y_{n}, \gamma x\right\} \vdash \delta M
$$

This deduction must be an instance of the given principal deduction for $M$, and hence $\left\langle\beta_{1}, \ldots, \beta_{n},\left(F_{\gamma} \delta\right)\right\rangle$ is an instance of $\left\langle\alpha_{1}, \ldots, \alpha_{n},(\mathbf{F} \xi \eta)\right\rangle$. Therefore by (18) these two sequences are trivial variants of one another. Now we construct a principal deduction with the same hypotheses and conclusion as (17). To do this, replace all type-variables in the deduction (16) which do not occur in

$$
\left\langle\beta_{1}, \ldots, \beta_{n},(\mathbf{F} \gamma \delta)\right\rangle
$$

by distinct new variables not occurring in $\left\langle\alpha_{1}, \ldots, \alpha_{n},(F \xi \eta)\right\rangle$, and then make the substitutions which change $\left\langle\beta_{1}, \ldots, \beta_{n},(\mathbf{F} \gamma \delta)\right\rangle$ to $\left\langle\alpha_{1}, \ldots, \alpha_{n},(\mathbf{F} \xi \eta)\right\rangle$. The result is a deduction giving (17), and it is principal because it is a trivial variant of (16).

COROllary 2.1. If the only ob-variables in $M$ are $x_{1}, \ldots, x_{n}(n \geqq 1)$, and

$$
\left\{\alpha_{1} x_{1}, \ldots, \alpha_{n} x_{n}\right\} \vdash_{p} \beta M,
$$


then

where

$$
\vdash_{p}\left(\mathbf{F}_{n} \alpha_{1} \cdots \alpha_{n} \beta\right)\left(\left[x_{1}, \ldots, x_{n}\right] . M\right),
$$

$$
\left(\boldsymbol{F}_{n} \alpha_{1} \cdots \alpha_{n} \beta\right) \quad \text { is } \quad \boldsymbol{F}_{\alpha_{1}}\left(\mathbf{F}_{\alpha_{2}}\left(\cdots\left(\boldsymbol{F}_{\left.\alpha_{n} \beta\right)} \beta\right)\right)\right.
$$

or just $\beta$ when $n=0$, and

$$
\left[x_{1}, \ldots, x_{n}\right] \cdot M \equiv\left[x_{1}\right] .\left(\left[x_{2}\right] .\left(\cdots\left(\left[x_{n}\right] \cdot M\right) \cdot \cdots\right)\right)
$$

or just $M$ when $n=0$.

Example 5. Define

$$
A \equiv[x, y, z] \cdot \mathbf{K}(x y)(x z):
$$

then by Example 2 and Corollary 2.1,

$$
\vdash_{p} \mathbf{F}(\mathbf{F} a b)(\mathbf{F} a(\mathbf{F} a b)) A .
$$

RemarK. H. Curry has pointed out that if the definition of $[x] . M$ included, as it usually does, the clause

$$
[x] .(U x) \equiv U \text { when } U \text { does not contain } x
$$

(and clause (iii) were only applied when (iv) was not applicable), then Theorem 2 would be false. For example the deduction

$$
\frac{F a b y \quad a x}{b(y x)}
$$

is a principal one for $(y x)$, and by (iv),

$$
[x] \cdot(y x) \equiv y,
$$

so Theorem 2 would say that

$$
\mathrm{F} a b y \vdash_{p} \mathrm{~F} a b y \text {, }
$$

which is false because the one-step deduction

$$
\text { Fab } y
$$

is not a principal one for $y$. The proof of Theorem 2 breaks down when (iv) is used because then the p.t.s. of $[x] . M$ may not be compound; but this does not mean that Theorem 2 is useless; see the proof of Theorem 3 later.

Incidentally, Theorem 2 can be proved by induction on the construction of $M$ without using (14) and (15); though this proof is longer than the above one, it does give an alternative proof of (15), because (15) follows from Theorems 1 and 2.

5. An alternative approach to typed combinators. Now besides the formal system set up in Definitions 1 to 4 , there is another way of introducing typerestrictions into combinatory logic. In Definition 1 , instead of postulating two basic combinators $\mathbf{S}$ and $\mathbf{K}$, we could postulate an infinite set of basic combinators. 
For each ordered triple $\alpha, \beta, \gamma$ of types there would be a distinct basic combinator $\mathbf{S}_{\alpha \beta \gamma}$, and for each pair $\alpha, \beta$ there would be a distinct basic $\mathbf{K}_{\alpha \beta}$; the axiom-schemes for $\mathbf{S}$ and $\mathbf{K}$ in Definition 4 would be replaced by the infinite set of axioms

$$
\begin{aligned}
& \mathbf{F}\left(F_{\alpha}(\mathbf{F} \beta \gamma)\right)\left(\mathbf{F}\left(F_{\alpha \beta}\right)\left(F_{\alpha \gamma}\right)\right) S_{\alpha \beta \gamma} \\
& F_{\alpha}(\mathbf{F} \beta \alpha) \mathbf{K}_{\alpha \beta} .
\end{aligned}
$$

The type-variables in Definition 2 would not be used at all.

It can be seen that each combinator in this alternative system would have just one type (if it had a type at all), in contrast to the infinite number of types given to each combinator in the former system. Hence it is easier to give a set-theoretic interpretation to the combinators in the alternative system than in the original system; for instance $\mathbf{K}_{\alpha \beta}$ is interpreted as the function $f$ such that (in the usual function notation)

$$
(f(x))(y)=x
$$

for each member $x$ of the set $\alpha$, and each member $y$ of $\beta$. This $f$ changes its argument $x$ into a constant-valued function. But in the former system, the basic combinator $\mathbf{K}$ did not represent a particular function $f$, but rather the abstract notion of forming a constant function from any object; this is much harder to interpret set-theoretically. For instance $\mathbf{K}$ can be applied to itself; [more precisely, $(\mathbf{K} \mathbf{K})$ is a combinator and it even has a p.t.s. $\mathbf{F} a(\mathbf{F} b(\mathbf{F} c b))]$ but the set-theoretic meaning of applying $\mathbf{K}$ to itself is not so clear. Actually the combinators perform their tasks without needing any such interpretation (compare the parentheses and punctuation symbols in the usual forms of predicate calculus), but if a set-theoretic interpretation is wanted, then it seems more convenient to use the system based on an infinity of combinators $\mathbf{S}_{\alpha \beta \gamma}, \mathbf{K}_{\alpha \beta}$ (see [5], for example).

However, most of the fundamental syntactical properties of the combinators in this alternative system are independent of the types of the particular $\mathbf{S}_{\alpha \beta \gamma}$ and $\mathbf{K}_{\alpha \beta}$ involved. So for discussing these fundamental properties the original system is more natural.

In the alternative system, reduction and equality are defined as in Definition 11, except that $\left(\mathbf{K}_{\alpha \beta} U V\right)$ may be replaced by $U$ only when

$$
\vdash \alpha U \text { and } \vdash \beta V \text {, }
$$

and $\left(\mathbf{S}_{\alpha \beta \gamma} U V W\right)$ may be replaced by $U W(V W)$ only when

$$
\vdash \mathbf{F} \alpha(\mathbf{F} \beta \gamma) U \text { and } \vdash \mathbf{F} \alpha \beta V \text { and } \vdash \alpha W \text {. }
$$

These conditions ensure that an ob with a type is replaced by another with the same type; hence if $X \geqq Y$ or $X=Y$, then $X$ and $Y$ both have the same type (and if either has a type, the other does).

Now it looks as if it might be possible to find a subsystem of the original system based on $\mathbf{S}$ and $\mathbf{K}$ to correspond to the infinite-based system, by using the fact 
that each ob with a type has a unique p.t.s. (neglecting trivial variants) and somehow making principal type-schemes in the original system do the work of types in the infinite-based system. An ob in the finite-based system whose p.t.s. is a type $\alpha$ can have only one type, since all its types are instances of $\alpha$, which contains no variables. Therefore we could try to set up a correspondence between infinite-based obs, and finite-based obs whose principal type-schemes are types. One way of doing this is as follows (letters " $X$ ", " $Y$ " will denote finite-based obs here, and " $M$ ", " $N$ ", " $P$ " infinite-based obs).

Firstly, suppose we could prove

$$
\begin{aligned}
& \text { If } \vdash \alpha X \text { in the system of } \S 1 \text {, then there is } \\
& \text { an ob } X_{\alpha} \text { such that } \vdash_{p} \alpha X_{\alpha} \text { and } X_{\alpha} \geqq X .
\end{aligned}
$$

Then applying (19) to $\mathbf{K}$ and $\mathbf{S}$ would give us, for each triple $\alpha, \beta, \gamma$ of types, two obs $\mathbf{K}_{\alpha \beta}^{*}$ and $\mathbf{S}_{\alpha \beta \gamma}^{*}$ with the principal type-schemes, respectively,

$$
F_{\alpha}(\mathbf{F} \beta \alpha), \quad F\left(F_{\alpha}(\mathbf{F} \beta \gamma)\right)\left(F(F \alpha \beta)\left(F_{\alpha \gamma}\right)\right) .
$$

These two type-schemes contain no variables, so they are the only types that $\mathbf{K}_{\alpha \beta}^{*}$ and $\mathbf{S}_{\alpha \beta \gamma}^{*}$ could have. Hence to each combinator $P$ (with a type $\delta$ ) in the infinitebased system, would correspond a finite-based ob $P^{T}$ whose only type was $\delta$; $P^{T}$ would be defined thus:

$$
\left(\mathbf{S}_{\alpha \beta \gamma}\right)^{T} \equiv \mathbf{S}_{\alpha \beta \gamma}^{*}, \quad\left(\mathbf{K}_{\alpha \beta}\right)^{T} \equiv \mathbf{K}_{\alpha \beta}^{*}, \quad(M N)^{T} \equiv\left(M^{T} N^{T}\right) .
$$

To complete the embedding of the infinite-based system in the finite-based one, we would only need to show that

(20) $M \geqq N$ in the infinite-based system iff $M^{T} \geqq N^{T}$ in the finite-based system.

Half of this equivalence follows from (19); in fact, to show that $M \geqq N$ implies $M^{T} \geqq N^{T}$, it is enough to show that for all $M, N, P$,

$$
\mathrm{S}_{\alpha \beta \gamma}^{*} M^{T} N^{T} P^{T} \geqq M^{T} P^{T}\left(N^{T} P^{T}\right), \quad \mathbf{K}_{\alpha \beta}^{*} M^{T} N^{T} \geqq M^{T}
$$

in the finite-based system. These are true, because

$$
\begin{aligned}
K_{\alpha \beta}^{*} M^{T} N^{T} & \geqq \mathbf{K} M^{T} N^{T} \quad \text { by }(19) \\
& \geqq M^{T} \quad \text { by Definition } 11,
\end{aligned}
$$

and similarly for $\mathbf{S}_{\alpha \beta \gamma}^{*}$.

The embedding can also be seen to be one-to-one, using the actual structure of $\mathbf{K}_{\alpha \beta}^{*}$ and $\mathbf{S}_{\alpha \beta \gamma}^{*}$ given by the proof of (19) in the next section.

However I do not know if the converse half of $(20)$ is true $\left({ }^{7}\right)$.

(7) The main use of the alternative system (with each atom having a unique type) is to represent certain sets $S$ of functionals (see [5] for example). We represent each $\phi$ in $S$ with type $\alpha$ by an ob $[\phi]$ with the same type, such that $[\phi][\psi]=[\phi(\psi)]$ if $\phi$ has type $F \beta \gamma$ and $\psi$ is in $S$ with type $\beta$ and $\phi(\psi)$ is in $S$. One purpose of the above embedding (suggested by Curry) is to show that every $\phi$ which can be represented as above can also be represented by a finite-based ob $[\phi]^{\prime}$ such that $[\phi]^{\prime}[\psi]^{\prime}=[\phi(\psi)]^{\prime}$. For this result we do not need the converse half of $(20)$; we simply define $[\phi]^{\prime}$ to be $[\phi]^{T}$. 
The proof of (19) will be given in the next section (Corollary 3.1). Although it is only used in the above argument in the case that $\alpha$ is a type, it seems easiest to prove it first for the case that $\alpha$ contains only variables, and then extend it to the other case. This extension assumes that each basic type is the p.t.s. of at least one ob (not necessarily an ob-atom).

6. Every type-scheme is a p.t.s. The main purpose of this section is to verify (19), which seems interesting in its own right, besides its application in the preceding section.

The obs of this section are those of $\$ 1-4$, not the alternative system in $\S 5$. Typevariables denoted by distinct letters will be assumed to be distinct, and calling a variable "new" will mean that it does not occur in any type-scheme mentioned earlier in the section.

LEMMA 7. Given any distinct type-variables $b, c, d, e$, define

$$
\begin{array}{ll}
\xi_{1} \equiv \mathbf{F}(\mathbf{F} d e) c & \eta_{1} \equiv \mathbf{F} d c \\
\xi_{2} \equiv \mathbf{F}(\mathbf{F} d e) c & \eta_{2} \equiv \mathbf{F e c} \\
\xi_{3} \equiv \mathbf{F} b(\mathbf{F} d e) & \eta_{3} \equiv \mathbf{F} b d \\
\xi_{4} \equiv \mathbf{F} b(\mathbf{F} d e) & \eta_{4} \equiv \mathbf{F} b e .
\end{array}
$$

Then there exist combinators $B_{1}, \ldots, B_{4}$ such that for $i=1, \ldots, 4$,

$$
\vdash_{p} \mathbf{F}\left(\mathbf{F} \xi_{i} \xi_{i}\right)\left(\mathbf{F}\left(\mathbf{F} \eta_{i} \eta_{i}\right)\left(\mathbf{F} \xi_{i} \xi_{i}\right)\right) B_{i} .
$$

Also there is a combinator $C$ such that

$$
\vdash_{p} \mathbf{F}(\mathbf{F} b b)(\mathbf{F}(\mathbf{F} c c)(\mathbf{F}(\mathbf{F} b c)(\mathbf{F} b c))) C .
$$

Proof. Tedious; see the end of the section.

THEOREM 3. If $X$ is an ob whose atoms are all given types by the axioms, and

$$
\vdash \alpha X
$$

for some $\alpha$ containing only variables, then there exists an ob $X_{\alpha}$ such that

$$
X_{\alpha} \geqq X \text { and } \vdash_{p} \alpha X_{\alpha} ;
$$

if $X$ is a combinator, then so is $X_{\alpha}$.

Proof. First suppose that for each $\alpha$ containing only variables there exists a combinator $V_{\alpha}$ such that

$$
\vdash_{p} F_{\alpha \alpha} V_{\alpha} \text {. }
$$

Then using the ob $A$ from Example 5, $\$ 4$, define

Then

$$
X_{\alpha} \equiv \mathbf{S}(A \mathbf{I}) V_{\alpha} X
$$

$$
\begin{aligned}
X_{\alpha} & \geqq A \mathbf{I} X\left(V_{\alpha} X\right) \\
& \geqq \mathbf{K}(\mathbf{I} X)\left(\mathbf{I}\left(V_{\alpha} X\right)\right) \subset \text { by }(14) \text { and the def. of } A \\
& \geqq X .
\end{aligned}
$$


Also we have by Examples 5 and 3,

$$
\vdash_{p} \mathbf{F}(\mathbf{F} a b)(\mathbf{F} a(\mathbf{F} a b)) A \quad \vdash_{p} \mathbf{F} a a \mathbf{I} .
$$

Therefore by Corollary 1.3, substituting $a$ for $b$,

$$
\vdash_{p} \mathrm{~F} a(\mathrm{~F} a a)(A \mathbf{I}) \text {. }
$$

Now by the axiom-schemes,

$$
\vdash_{p} \mathbf{F}(\mathbf{F} a(\mathbf{F} b c))(\mathbf{F}(\mathbf{F} a b)(\mathbf{F} a c)) \mathbf{S} .
$$

Hence by Corollary 1.3, substituting $a$ for $b$ and $c$,

$$
\vdash_{p} \mathbf{F}(\mathbf{F} a a)(\mathbf{F} a a)(\mathbf{S}(A \mathbf{I})) \text {. }
$$

Therefore by Corollary 1.3 again, substituting $\alpha$ for $a$,

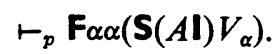

Now by Theorem $1 X$ has a p.t.s. $\alpha_{0}$, and $\alpha$ is an h.c.i. of $\alpha$ and $\alpha_{0}$, so by Corollary 1.3,

$$
\vdash_{p} \alpha\left(\mathbf{S}(A \mathbf{I}) V_{\alpha} X\right) \text {. }
$$

It only remains to prove the existence of $V_{\alpha}$ for all $\alpha$; this is done by induction on the number of occurrences of $F$ in $\alpha$ (called the "length" of $\alpha$ ).

Basis. If $\alpha$ is a variable, choose $V_{\alpha} \equiv \mathbf{I}$.

Induction step. Suppose $\alpha$ is $\mathbf{F} \beta \gamma$ for some $\beta, \gamma$. We shall construct $V_{\alpha}$ by induction on the number of variables common to $\beta$ and $\gamma$.

Basis. Suppose there are no such variables. By the hypothesis of the induction on $\alpha$ we have

$$
\vdash_{p} \mathrm{~F} \beta \beta V_{\beta}, \quad \vdash_{p} \mathrm{~F}_{\gamma \gamma} V_{\gamma} .
$$

Then using the $C$ from Lemma 7, define

$$
V_{\alpha} \equiv C V_{\beta} V_{\gamma}
$$

By Lemma 7, for new variables $b$ and $c$,

$$
\vdash_{p} \mathbf{F}(\mathbf{F} b b)(\mathbf{F}(\mathbf{F} c c)(\mathbf{F}(\mathbf{F} b c)(\mathbf{F} b c))) C .
$$

Hence by Corollary 1.3, substituting $\beta$ for $b$,

$$
\vdash_{p} \mathbf{F}(\mathbf{F} c c)(\mathbf{F}(\mathbf{F} \beta c)(\mathbf{F} \beta c))\left(C V_{\beta}\right) \text {. }
$$

Now an h.c.i. of $F_{c c}$ and $F_{\gamma \gamma}$ is $F_{\gamma \gamma}$, so by Corollary 1.3 again, substituting $\gamma$ for $c$,

$$
\vdash_{p} \mathbf{F}(\mathbf{F} \beta \gamma)(\mathbf{F} \beta \gamma)\left(C V_{\beta} V_{y}\right)
$$

as required. Notice that if a variable was common to $\beta$ and $\gamma, \mathbf{F}_{\gamma \gamma}$ would not satisfy the conditions for $\xi^{*}$ in Corollary 1.3 .

Induction step. Suppose there is a variable $h$ which occurs in both $\beta$ and $\gamma$. Let $g$ be a new variable, and define

$$
\beta^{\prime} \equiv[g / h] \beta .
$$


Then $\beta^{\prime}$ has one less variable in common with $\gamma$ than $\beta$ has, and

$$
\beta \equiv[h / g] \beta^{\prime} .
$$

By the induction hypothesis there is a $V$ such that

$$
\vdash_{p} \mathbf{F}\left(\mathbf{F} \beta^{\prime} \gamma\right)\left(\mathbf{F} \beta^{\prime} \gamma\right) V
$$

We shall now construct a $V_{\alpha}$ such that

$$
\vdash_{p} \mathbf{F}(\mathbf{F} \beta \gamma)(\mathbf{F} \beta \gamma) V_{\alpha}
$$

Case 1. When both $\beta$ and $\gamma$ are variables, then $\beta \equiv \gamma \equiv h$ and we need

$$
\vdash_{p} F(F h h)(F h h) V_{\alpha} \text {. }
$$

Define $V_{\alpha}$ to be $\mathbf{S}(A \mathbf{I})$ and use (21).

Case 2. When $\beta$ is compound, say $\beta \equiv \mathbf{F} \delta \varepsilon$, and $h$ occurs in $\delta$. Then $\beta^{\prime} \equiv \mathbf{F} \delta^{\prime} \varepsilon^{\prime}$ and $g$ occurs in $\delta^{\prime}$ (but not in $\gamma$ ). Now $\mathbf{F} \delta \gamma$ is shorter than $\alpha$ because $\alpha$ is $\mathbf{F}(\mathbf{F} \delta \varepsilon) \gamma$, so by the hypothesis of the induction on $\alpha$ there exists $V_{1}$ such that

Also by (22),

$$
\vdash_{p} \mathbf{F}(\mathbf{F} \delta \gamma)(\mathbf{F} \delta \gamma) V_{1}
$$

$$
\vdash_{p} \mathbf{F}\left(\mathbf{F}\left(\mathbf{F} \delta^{\prime} \varepsilon^{\prime}\right) \gamma\right)\left(\mathbf{F}\left(\mathbf{F} \delta^{\prime} \varepsilon^{\prime}\right) \gamma\right) V .
$$

Now by Lemma 7 , where $\xi_{1}$ is $\mathbf{F}(\mathbf{F} d e) c$ for new variables $c, d, e$,

$$
\vdash_{p} \mathbf{F}\left(\mathbf{F} \xi_{1} \xi_{1}\right)\left(\mathbf{F}(\mathbf{F}(\mathbf{F} d c)(\mathbf{F} d c))\left(\mathbf{F} \xi_{1} \xi_{1}\right)\right) B_{1} .
$$

Define $V_{\alpha} \equiv B_{1} V V_{1}$.

Substituting $\delta^{\prime}, \varepsilon^{\prime}, \gamma$ for $d, e, c$ changes $\xi_{1}$ into $\mathbf{F}\left(\mathbf{F} \delta^{\prime} \varepsilon^{\prime}\right) \gamma$ which is $\mathbf{F} \beta^{\prime} \gamma$. Hence by Corollary 1.3,

$$
\vdash_{p} \mathbf{F}\left(\mathbf{F}\left(\mathbf{F} \delta^{\prime} \gamma\right)\left(\mathbf{F} \delta^{\prime} \gamma\right)\right)\left(\mathbf{F}\left(\mathbf{F} \beta^{\prime} \gamma\right)\left(\mathbf{F} \beta^{\prime} \gamma\right)\right)\left(B_{1} V\right)
$$

Now $\delta \equiv[h / g] \delta^{\prime}$ and $g$ does not occur in $\gamma$, so $\mathbf{F}(\mathbf{F} \delta \gamma)(\mathbf{F} \delta \gamma)$ is an instance of $\mathbf{F}\left(\mathbf{F} \delta^{\prime} \gamma\right)\left(\mathbf{F} \delta^{\prime} \gamma\right)$. Also every variable in the former type-scheme occurs in the latter one (since $h$ occurs in $\gamma$ ). Therefore Corollary 1.3 can be applied to $B_{1} V$ and $V_{1}$, with

Thus we obtain

$$
\begin{aligned}
\xi^{*} & \equiv \zeta \equiv \mathbf{F}(\mathbf{F} \delta \gamma)(\mathbf{F} \delta \gamma) \\
\eta^{*} & \equiv \mathbf{F}(\mathbf{F} \beta \gamma)(\mathbf{F} \beta \gamma)
\end{aligned}
$$

as required.

$$
\vdash_{p} \mathbf{F}(\mathbf{F} \beta \gamma)(\mathbf{F} \beta \gamma)\left(B_{1} V V_{1}\right)
$$

Case 3. When $\beta$ is compound, say $\beta \equiv \mathbf{F} \delta \varepsilon$, and $h$ occurs in $\varepsilon$ but not in $\delta$. Then $\beta^{\prime}$ is $\mathbf{F} \delta \varepsilon^{\prime}$ and $g$ occurs in $\varepsilon^{\prime}$. Now $\mathbf{F}_{\varepsilon \gamma}$ is shorter than $\alpha$, so by hypothesis of the induction on $\alpha$ there exists $V_{2}$ such that

Define $V_{\alpha} \equiv B_{2} V V_{2}$.

$$
\vdash_{p} \mathbf{F}\left(\boldsymbol{F}_{\varepsilon \gamma}\right)\left(\boldsymbol{F}_{\varepsilon \gamma}\right) V_{2} .
$$


By Lemma 7, where $\xi_{2}$ is $\mathbf{F}(\mathbf{F} d e) c$ for new variables $d, e, c$,

$$
\vdash_{p} \mathbf{F}\left(\mathbf{F} \xi_{2} \xi_{2}\right)\left(\mathbf{F}(\mathbf{F}(\mathbf{F} e c)(\mathbf{F} e c))\left(\mathbf{F} \xi_{2} \xi_{2}\right)\right) B_{2} .
$$

Therefore by Corollary 1.3 , substituting $\delta, \varepsilon^{\prime}, \gamma$ for $d, e, c$,

$$
\vdash_{p} \mathbf{F}\left(\mathbf{F}\left(\mathbf{F} \varepsilon^{\prime} \gamma\right)\left(\boldsymbol{F}_{\varepsilon^{\prime}} \gamma\right)\right)\left(\mathbf{F}\left(\mathbf{F} \beta^{\prime} \gamma\right)\left(\mathbf{F} \beta^{\prime} \gamma\right)\right)\left(B_{2} V\right) \text {. }
$$

Similarly to Case 2, $\mathbf{F}\left(\mathbf{F}_{\varepsilon \gamma}\right)\left(\mathbf{F}_{\varepsilon} \gamma\right)$ is an instance of $\mathbf{F}\left(\mathbf{F}_{\varepsilon^{\prime}} \gamma\right)\left(\mathbf{F}_{\varepsilon^{\prime} \gamma}\right)$ and every variable in the former also occurs in the latter. Therefore by Corollary 1.3, substituting $h$ for $g$,

as required.

$$
\vdash_{p} \mathbf{F}(\mathbf{F} \beta \gamma)(\mathbf{F} \beta \gamma)\left(B_{2} V V_{2}\right)
$$

Case 4. When $\gamma$ is compound, say $\gamma \equiv \mathbf{F} \delta \varepsilon$, with $h$ occurring in $\delta$. The scheme $\mathbf{F} \beta \delta$ is shorter than $\alpha$, so we have a $V_{3}$ such that

Also by (22),

$$
\vdash_{p} \mathbf{F}(\mathbf{F} \beta \delta)(\mathbf{F} \beta \delta) V_{3} \text {. }
$$

Define $V_{\alpha} \equiv B_{3} V V_{3}$.

$$
\vdash_{p} \mathbf{F}\left(\mathbf{F} \beta^{\prime}(\mathbf{F} \delta \varepsilon)\right)\left(\mathbf{F} \beta^{\prime}(\mathbf{F} \delta \varepsilon)\right) V
$$

By Lemma 7, where $\xi_{3}$ is $\mathbf{F} b(\mathbf{F} d e)$ for new variables $b, d, e$,

$$
\vdash_{p} \mathbf{F}\left(\mathbf{F} \xi_{3} \xi_{3}\right)\left(\mathbf{F}(\mathbf{F}(\mathbf{F} b d)(\mathbf{F} b d))\left(\mathbf{F} \xi_{3} \xi_{3}\right)\right) B_{3} .
$$

Therefore by Corollary 1.3 , substituting $\beta, \delta, \varepsilon$ for $b, d, e$,

$$
\vdash_{p} \mathbf{F}\left(\mathbf{F}\left(\mathbf{F} \beta^{\prime} \delta\right)\left(\mathbf{F} \beta^{\prime} \delta\right)\right)\left(\mathbf{F}\left(\mathbf{F} \beta^{\prime} \gamma\right)\left(F \beta^{\prime} \gamma\right)\right)\left(B_{3} V\right) .
$$

And as in the previous cases we get

$$
\vdash_{p} \mathbf{F}(\mathbf{F} \beta \gamma)(\mathbf{F} \beta \gamma)\left(B_{3} V V_{3}\right)
$$

by substituting $h$ for $g$ and using Corollary 1.3.

Case 5. When $\gamma$ is $\mathbf{F} \delta \varepsilon$ and $h$ occurs in $\varepsilon$ but not in $\delta$. Then $\mathbf{F} \beta \varepsilon$ is shorter than $\alpha$, so we can get a $V_{4}$ such that

Define $V_{\alpha} \equiv B_{4} V V_{4}$.

$$
\vdash_{p} F(F \beta \varepsilon)(F \beta \varepsilon) V_{4}
$$

By Lemma 7, where $\xi_{4}$ is $\mathbf{F} b(\mathbf{F} d e)$ for new variables $b, d, e$,

$$
\vdash_{p} \mathbf{F}\left(\mathbf{F} \xi_{4} \xi_{4}\right)\left(\mathbf{F}(\mathbf{F}(\mathbf{F} b e)(\mathbf{F} b e))\left(\mathbf{F} \xi_{4} \xi_{4}\right)\right) B_{4} .
$$

As in Case 4, two applications of Corollary 1.3 give

$$
\vdash_{p} F(F \beta \gamma)(F \beta \gamma)\left(B_{4} V V_{4}\right),
$$

completing the proof of Theorem 3 .

COROLlaRY 3.1. The theorem remains true if $\alpha$ contains basic types, provided that to each basic type $\theta$ corresponds at least one ob $Z_{\theta}$ (not necessarily an atom) such that $\vdash_{p} \theta Z_{\theta}$. 
Proof. It is enough to prove the existence of a $V_{\alpha}$ for the case when $\alpha$ contains basic types. Let $\theta_{1}, \ldots, \theta_{n}$ be the basic types in $\alpha$, and let $\alpha_{0}$ be the result of substituting a distinct new type-variable $a_{i}$ for each $\theta_{i}$ in $\alpha$. Then $\alpha$ will be the result of substituting $\theta_{1}, \ldots, \theta_{n}$ for $a_{1}, \ldots, a_{n}$ in $\alpha_{0}$. By a nonprincipal deduction obtained by substituting $\alpha_{0}$ for $a$ in Example $1, \S 1$,

$$
-\mathbf{F}_{\alpha_{0} \alpha_{0}} \mathbf{I} \text {. }
$$

Now by the axiom-scheme for $\mathbf{K}$,

so by Rule (F),

$$
\vdash \mathbf{F}\left(\mathbf{F} \alpha_{0} \alpha_{0}\right)\left(\mathbf{F} a_{n}\left(\mathbf{F} \alpha_{0} \alpha_{0}\right)\right) \mathbf{K}
$$

$$
\vdash F a_{n}\left(\mathbf{F} \alpha_{0} \alpha_{0}\right)(\mathbf{K I}) .
$$

Repeating this argument, we get

$$
\vdash\left(\mathbf{F}_{n} a_{1} \cdots a_{n}\left(\mathbf{F} \alpha_{0} \alpha_{0}\right)\right)(\mathbf{K}(\mathbf{K}(\cdots(\mathbf{K} \mathbf{I}) \cdots))) .
$$

Therefore by Theorem 3 there exists a combinator $U$ such that

$$
\vdash_{p}\left(\mathbf{F}_{n} a_{1} \cdots a_{n}\left(\mathbf{F}_{\alpha_{0} \alpha_{0}}\right)\right) U
$$

Now by the assumption, there exist obs $Z_{1}, \ldots, Z_{n}$ such that for each $i$,

$$
\vdash_{p} \theta_{i} Z_{i}
$$

Therefore by Corollary 1.3 used $n$ times,

$$
\vdash_{p} F_{\alpha \alpha}\left(U Z_{1} \cdots Z_{n}\right)
$$

so we can choose $V_{\alpha}$ to be $\left(U Z_{1} \cdots Z_{n}\right)$, completing the proof.

REMARK. Equality and types. It might at first sight be hoped that if $X \geqq Y$, then $X$ and $Y$ would have the same principal type-scheme. Theorem 3 shows that this is false, and the following example of Curry's,

$$
\text { SIII } \geqq I I(I I)
$$

shows that $X$ does not need to have a type at all, even though $Y$ has types. All that we can say is that if $X \geqq Y$ and $X$ has types, then by (13), the p.t.s. of $X$ is an instance of that of $Y$.

When $X=Y$ in general, the situation is even less tidy; in fact there exist such $X$ and $Y$ which have no type-schemes in common at all. For example, define

$$
\alpha \equiv \mathbf{F} c(\mathbf{F} c c), \quad \beta \equiv \mathbf{F}(\mathbf{F} d d) d ;
$$

then by Theorem 3 there exist combinators $I_{1}$ and $I_{2}$ such that

$$
\begin{aligned}
I_{1} \geqq \mathbf{I}, & I_{2} \geqq \mathbf{I}, \\
\vdash_{p} \text { F } \alpha \alpha I_{1}, & \vdash_{p} \text { F } \beta \beta I_{2} .
\end{aligned}
$$


Hence $I_{1}=I_{2}$, but any type-scheme possessed by both $I_{1}$ and $I_{2}$ would have to have both the forms

$$
\mathbf{F}\left(\mathbf{F}_{\gamma}\left(\mathbf{F}_{\gamma \gamma}\right)\right)\left(\mathbf{F}_{\gamma}\left(\mathbf{F}_{\gamma \gamma}\right)\right), \quad \mathbf{F}(\mathbf{F}(\mathbf{F} \delta \delta) \delta)(\mathbf{F}(\mathbf{F} \delta \delta) \delta),
$$

which is impossible.

Proof of Lemma 7. The following is one way of constructing the combinators $B_{1}, \ldots, B_{4}, C$.

First define

$$
D \equiv[x] \cdot \mathbf{K I}(A x)
$$

Then

$$
\vdash_{p} \mathbf{F}(\mathbf{F} a b)(\mathbf{F} c c) D .
$$

Proof. By Example 5, $\$ 4$, we have

$$
\vdash_{p} \mathbf{F}(\mathbf{F} a b)(\mathbf{F} a(\mathbf{F} a b)) A .
$$

A one-step principal deduction for $x$ gives us

$$
d x \vdash_{p} d x .
$$

Now an h.c.i. of $d$ and F $a b$ is Fab itself, so by Corollary 1.4, substituting Fab for $d$ and combining the above two deductions gives

$$
\mathbf{F} a b \times \vdash_{p} \mathbf{F} a(\mathbf{F} a b)(A x) .
$$

By the axiom-schemes and Example 3,

$$
\vdash_{p} \mathbf{F} a(\mathbf{F} d a) \mathbf{K}, \quad \vdash_{p} \mathbf{F} c c \mathbf{l},
$$

so by Corollary 1.3, substituting F $c c$ for $a$,

$$
\vdash_{p} \mathbf{F} d(\mathbf{F} c c)(\mathbf{K} \mathbf{I}) .
$$

An h.c.i. of $d$ and $\mathbf{F} a(\mathbf{F} a b)$ is $\mathbf{F} a(\mathbf{F} a b)$, so by Corollary 1.4, substituting $\mathbf{F} a(\mathbf{F} a b)$ for $d$ in (24),

$$
\mathbf{F} a b x \vdash_{p} \mathbf{F} c c(\mathbf{K I}(A x)) .
$$

Finally Theorem 2 gives the result.

Next define $E \equiv[x, y] . D(A \mathbf{I}(\mathbf{K} y) x) y$. Then

$$
\vdash_{p} \mathbf{F}(\mathbf{F} a b)(\mathbf{F} b b) E \text {. }
$$

Proof. First of all, we have

$$
\begin{aligned}
& \vdash_{p} \mathbf{F} b(\mathbf{F} a b) \mathbf{K}, \\
& b y \vdash_{p} b y \text {. }
\end{aligned}
$$

Therefore by Corollary 1.5 ,

$$
b y \vdash_{p} \mathbf{F} a b(\mathbf{K} y) \text {. }
$$


By the start of the proof of Theorem 3,

$$
\vdash_{p} \mathbf{F} c(\mathbf{F} c c)(A \mathbf{l}) .
$$

Therefore by Corollary 1.4, substituting Fab for $c$,

$$
b y \vdash_{p} \mathbf{F}(\mathbf{F} a b)(\mathbf{F} a b)(A \mathbf{l}(\mathbf{K} y)) \text {. }
$$

Now $c x \vdash_{p} c x$, so by Corollary 1.4 , substituting Fab for $c$,

$$
\mathbf{F} a b x, b y \vdash_{p} \mathbf{F} a b(A \mathbf{l}(\mathbf{K} y) x) .
$$

Therefore by (23) and Corollary 1.5,

$$
\mathbf{F} a b x, b y \vdash_{p} \mathbf{F} c c(D(A \mathbf{I}(\mathbf{K} y) x)) .
$$

Now for a new variable $e$,

$$
e y \vdash_{p} e y .
$$

To combine the above two deductions by the proof of Theorem 1 we need an h.c.i. of the two sequences

$$
\langle b, \mathbf{F} c c\rangle \quad\langle e, \mathbf{F} e g\rangle .
$$

A suitable h.c.i. is $\langle b, \mathbf{F} b b\rangle$, so we can substitute $b$ for $c$ and $e$ in the two deductions and combine them to get

$$
\mathbf{F} a b x, b y \vdash_{p} b(D(A \mathbf{I}(\mathbf{K} y) x) y) .
$$

The result follows by Corollary 2.1 .

Define $G \equiv[x, y] . A \mathbf{l}(\mathbf{K} y) x$. Then Corollary 2.1 applied to (27) gives us

$$
\vdash_{p} \mathbf{F}(\mathbf{F} a b)(\mathbf{F} b(\mathbf{F} a b)) G .
$$

Define $H \equiv[u, x, y, z] . \mathbf{K}(\mathbf{K} z)(G(\mathbf{S K}) y x)(E x(u z))$. Then

$$
\vdash_{p} \mathbf{F}(\mathbf{F} a c)(\mathbf{F}(\mathbf{F} b c)(\mathbf{F}(\mathbf{F} b b)(\mathbf{F} a a))) H .
$$

Proof. By the proof of Example 3, §3,

$$
\vdash_{p} \mathbf{F}(\mathbf{F} b c)(\mathbf{F} b b)(\mathbf{S K}) .
$$

Therefore by Corollary 1.3, substituting F $b c$ for $a$ and F $b b$ for $b$ in (28),

$$
\vdash_{p} \mathbf{F}(\mathbf{F} b b)(\mathbf{F}(\mathbf{F} b c)(\mathbf{F} b b))(G(\mathbf{S K})) .
$$

Now $d y \vdash_{p} d y$. Therefore by Corollary 1.4, substituting Fbb for $d$,

Similarly,

$$
\mathbf{F} b b \quad y \vdash_{p} \mathbf{F}(\mathbf{F} b c)(\mathbf{F} b b)(G(\mathbf{S K}) y) .
$$

$$
\mathbf{F} b b y, \mathbf{F} b c x \vdash_{p} \mathbf{F} b b(G(\mathbf{S K}) y x) .
$$

Now by (25),

$$
\vdash_{p} \mathbf{F}(\mathbf{F} b c)(\mathbf{F} c c) E
$$


Therefore by Corollary 1.4 ,

$$
\mathbf{F} b c x \vdash_{p} \mathbf{F} c c(E x) \text {. }
$$

Also Fac $u, a z \vdash_{p} c(u z)$. Therefore by Corollary 1.5,

$$
\text { Fbc } x, \text { Fac } u, a z \vdash_{p} c(\operatorname{Ex}(u z)) \text {. }
$$

Now for new variables $a, d, e$, we have

$$
\begin{gathered}
\vdash_{p} \mathbf{F} a(\mathbf{F} d a) \mathbf{K}, \\
a z \vdash_{p} a z .
\end{gathered}
$$

Therefore by Corollary 1.5 ,

$$
a z \vdash_{p} \mathbf{F d a}(\mathbf{K z}) \text {. }
$$

Also $\vdash_{p} \mathbf{F} b(\mathbf{F} e b) \mathbf{K}$. Therefore by Corollary 1.4, substituting Fda for $b$,

$$
a z \vdash_{p} \mathbf{F} e(\mathbf{F} d a)(\mathbf{K}(\mathbf{K z})) \text {. }
$$

To combine this with (30) by the proof of Theorem 1 we need an h.c.i. of

$$
\mathbf{F e}(\mathbf{F} d a), \quad \mathbf{F}(\mathbf{F} b b) g .
$$

A suitable h.c.i. is $\mathbf{F}(\mathbf{F} b b)(\mathbf{F} d a)$, since all its variables occur in $\mathbf{F} b b$ or in $\mathbf{F e}(\mathbf{F} d a)$; then substituting $\mathrm{F} b b$ for $e$ in the above deduction and combining with (30) gives

$$
\mathbf{F} b b y, \mathbf{F} b c x, a z \vdash_{p} \mathbf{F} d a(\mathbf{K}(\mathbf{K z})(G(\mathbf{S K}) y x)) .
$$

To combine this with (31) by the proof of Theorem 1 we need an h.c.i. of

$$
\langle\mathbf{F} b c, a, \mathbf{F} d a\rangle \text { and }\langle\mathbf{F} b c, a, \mathbf{F} c \boldsymbol{g}\rangle \text {. }
$$

A suitable h.c.i. is obtained by substituting $c$ for $d$ and $a$ for $g$; making the same substitution in the above deduction and combining with (31) gives

$$
\mathbf{F} a c u, \mathbf{F} b b y, \mathbf{F} b c x, a z \vdash_{p} a(\mathbf{K}(\mathbf{K} z)(G(\mathbf{S K}) y x)(E x(u z))) \text {. }
$$

The result follows by Corollary 2.1 .

Now suppose that for each $i=1, \ldots, 4$ there exist two combinators $L_{i}$ and $M_{i}$ and a type-scheme $\gamma_{i}$ such that

$$
\vdash_{p} F \xi_{i} \gamma_{i} L_{i}, \quad \vdash_{p} F_{\eta_{i}} \gamma_{i} M_{i}
$$

and $\gamma_{i}$ contains all the variables in $\eta_{i}$. Then we can define

$$
B_{i} \equiv G\left(H L_{i} M_{i}\right)
$$

This has the required p.t.s., namely

$$
\vdash_{p} \mathbf{F}\left(\mathbf{F} \xi_{i} \xi_{i}\right)\left(\mathbf{F}\left(\boldsymbol{F}_{\eta_{i} \eta_{i}}\right)\left(\mathbf{F} \xi_{i} \xi_{i}\right)\right) B_{i} .
$$

Proof. By Corollary 1.3, substituting $\xi_{i}$ for $a$ and $\gamma_{i}$ for $c$ in (29) and assuming that the $b$ therein is a new variable not occurring in $\xi_{i}$ or $\gamma_{i}$

$$
\vdash_{p} \mathbf{F}\left(\mathbf{F} b \gamma_{i}\right)\left(\mathbf{F}(\mathbf{F} b b)\left(\mathbf{F} \xi_{i} \xi_{i}\right)\right)\left(H L_{i}\right) \text {. }
$$


Now an h.c.i. of $\boldsymbol{F} b \gamma_{i}$ and $\boldsymbol{F} \eta_{i} \gamma_{i}$ is $\boldsymbol{F}_{\eta_{i}} \gamma_{i}$, and this satisfies the conditions of Corollary 1.3 since every variable in $\eta_{t}$ is in $\gamma_{t}$, so by that corollary,

$$
\vdash_{p} \mathbf{F}\left(\mathbf{F}_{\eta_{i} \eta_{i}}\right)\left(\mathbf{F} \xi_{i} \xi_{i}\right)\left(H L_{i} M_{i}\right) \text {. }
$$

Then the result follows by (28) and Corollary 1.3, substituting $\boldsymbol{F}_{\eta_{i}} \eta_{i}$ for $a$ and $\mathbf{F} \xi_{i} \xi_{i}$ for $b$ in (28).

Thus to construct $B_{1}, \ldots, B_{4}$, it only now remains to construct $L_{i}$ and $M_{i}$ for each $i$. When $i=1$ : let $\gamma_{1}$ be $\mathbf{F}(\mathbf{F}$ de $)(\mathbf{F} c c)$ and define

Then

$$
\begin{aligned}
L_{1} & \equiv[x, y, z] \cdot A \mathbf{I}([u] \cdot D u z) x y \\
M_{1} & \equiv[x, y] \cdot E([z] \cdot \mathbf{K I}(y z)(x z)) .
\end{aligned}
$$

and

$$
\vdash_{p} \mathbf{F}(\mathbf{F}(\mathbf{F} d e) c)(\mathbf{F}(\mathbf{F} d e)(\mathbf{F} c c)) L_{1}
$$$$
\vdash_{p} \mathbf{F}(\mathbf{F} d c)(\mathbf{F}(\mathbf{F} d e)(\mathbf{F} c c)) M_{1} .
$$

Proof. For $L_{1}$; by (23),

$$
\vdash_{p} \mathbf{F}(\mathbf{F} d e)(\mathbf{F} c c) D \text {. }
$$

Also $a u \vdash_{p} a u$. Therefore by Corollary 1.4, substituting Fde for $a$,

$$
\mathbf{F} \text { de } u \vdash_{p} \operatorname{Fcc}(D u) \text {. }
$$

Hence by Corollary 1.5,

$$
c z, \text { Fde } u \vdash_{p} c(D u z)
$$

Therefore by Theorem 2,

$$
c z \vdash_{p} \mathbf{F}(\mathbf{F} d e) c([u] . D u z)
$$

Now by (26),

$$
\vdash_{p} F a(F a a)(A I),
$$

so by Corollary 1.4 , substituting $\mathbf{F}(\mathbf{F} d e) c$ for $a$,

$$
c z \vdash_{p} \mathbf{F}(\mathbf{F}(\mathbf{F} d e) c)(\mathbf{F}(\mathbf{F} d e) c)(A \mathbf{l}([u] . D u z)) .
$$

Then using Corollary 1.4 twice we get

$$
\mathbf{F}(\mathbf{F} d e) c x, \mathbf{F} d e y, c z \vdash_{p} c(A \mathbf{I}([u] . D u z) x y)
$$

and the result for $L_{1}$ follows by Corollary 2.1 .

For $M_{1}$; by (24) we have $\vdash_{p} F e(F c c)(K I)$. Also

$$
\text { Fde } y, d z \vdash_{p} e(y z) \text {. }
$$

Therefore by Corollary 1.5 ,

$$
\mathbf{F} d e y, d z \vdash_{p} \mathbf{F} c c(\mathbf{K I}(y z)) .
$$

Similarly, by Corollary 1.5 again,

$\mathbf{F} d c x, \mathbf{F} d e y, d z \vdash_{p} c(\mathbf{K I}(y z)(x z))$. 
Therefore by Theorem 2 ,

$\mathbf{F} d c x, \mathbf{F} d e y r_{p} \mathbf{F} d c([z] \cdot \mathbf{K I}(y z)(x z))$.

Now by (25),

so by Corollary 1.5 ,

$$
\vdash_{p} \mathbf{F}(\mathbf{F} d c)(\mathbf{F} c c) E
$$

$$
\mathbf{F} d c x \text {, Fde } y \vdash_{p} \mathbf{F} c c(E([z] \cdot \mathbf{K I}(y z)(x z)))
$$

and the result for $M_{1}$ follows by Corollary 2.1.

When $i=2$; let $\gamma_{2}$ be $\mathbf{F e}(\mathbf{F} c c)$ and define

Then

$$
\begin{aligned}
L_{2} & \equiv[x, y] \cdot A \mathbf{I}(x(\mathbf{K} y)), \\
M_{2} & \equiv[x, y] \cdot A \mathbf{I}(x y) .
\end{aligned}
$$

and

$$
\vdash_{p} \mathbf{F}(\mathbf{F}(\mathbf{F} d e) c)(\mathbf{F} e(\mathbf{F} c c)) L_{2}
$$

$$
\vdash_{p} \mathbf{F}(\mathbf{F e c})(\mathbf{F e}(\mathbf{F} c c)) M_{2}
$$

Proof. For $L_{2}$; we have

therefore by Corollary 1.5,

$$
\begin{gathered}
e y \vdash_{p} e y, \\
\vdash_{p} \mathbf{F} e(\mathbf{F} d e) \mathbf{K}
\end{gathered}
$$

$$
e y r_{p} \mathbf{F} \operatorname{de}(\mathbf{K} y)
$$

Now

$$
a x \vdash_{p} a x \text {; }
$$

to combine these two deductions by the proof of Theorem 1 we need an h.c.i. of $a$ and $\mathbf{F}(\mathbf{F}$ de $) g$; since $\mathbf{F}(\mathbf{F}$ de $) c$ itself is a suitable h.c.i., substituting $\mathbf{F}(\mathbf{F} d e) c$ for $a$ and applying Rule (F) gives us

$$
\mathbf{F}(\mathbf{F} d e) c x, \text { e } y \vdash_{p} c(x(\mathbf{K} y)) .
$$

Therefore by (26) and Corollary 1.5 ,

$$
\mathbf{F}(\mathbf{F} d e) c x, e y \vdash_{p} \mathbf{F} c c(A \mathbf{l}(x(\mathbf{K} y)))
$$

and the result for $L_{2}$ follows by Corollary 2.1 .

For $M_{2}$; we have

$$
\text { Fec } x, \text { e } y \vdash_{p} c(x y) \text {. }
$$

Therefore by (26) and Corollary 1.5 ,

$$
\text { Fec } x, \text { e } y \vdash_{p} \mathbf{F} c c(A \mathbf{l}(x y))
$$

and the result follows by Corollary 2.1.

When $i=3$; let $\gamma_{3}$ be $\mathbf{F} b(\mathbf{F} d d)$ and define

$$
L_{3} \equiv[x, y] . \mathbf{S K}(x y), \quad M_{3} \equiv M_{2} \text {. }
$$


Then

and

$$
\vdash_{p} \mathbf{F}(\mathbf{F} b(\mathbf{F} d e))(\mathbf{F} b(\mathbf{F} d d)) L_{3}
$$

$$
\vdash_{p} \mathbf{F}(\mathbf{F} b d)(\mathbf{F} b(\mathbf{F} d d)) M_{3} .
$$

Proof. For $M_{3}$, use the result for $M_{2}$.

For $L_{3}$; by the proof of Example 3, §3,

Also

$$
\vdash_{p} \mathbf{F}(\mathbf{F} d e)(\mathbf{F} d d)(\mathbf{S K}) .
$$

$$
\mathbf{F} b c x, b y \vdash_{p} c(x y) \text {. }
$$

To combine these two deductions by the proof of Theorem 1, notice that $\mathbf{F}(\mathbf{F} d e)(\mathbf{F} d d)$ is a suitable h.c.i. of $\mathbf{F}(\mathbf{F} d e)(\mathbf{F} d d)$ and $\mathbf{F} c g$, and substitute $\mathbf{F}$ de for $c$, giving

$$
\mathbf{F} b(\mathbf{F} d e) x, b y \vdash_{p} \mathbf{F} d d(\mathbf{S K}(x y)) .
$$

The result for $L_{3}$ follows by Corollary 2.1 .

When $i=4$; let $\gamma_{4}$ be $\mathbf{F b}(\mathbf{F e e})$ and define

Then

$$
L_{4} \equiv[x, y] . E(x y), \quad M_{4} \equiv M_{2} .
$$

and

$$
\vdash_{p} \mathbf{F}(\mathbf{F} b(\mathbf{F} d e))(\mathbf{F} b(\mathbf{F} e e)) L_{4}
$$

Proof. For $L_{4}$;

$$
\vdash_{p} \mathbf{F}(\mathbf{F} b e)(\mathbf{F} b(\mathbf{F} e e)) M_{4}
$$

$$
\mathbf{F} b c x, b y \vdash_{p} c(x y)
$$

and by (25),

$$
\vdash_{p} \mathbf{F}(\mathbf{F} d e)(\mathbf{F} e e) E \text {. }
$$

By the proof of Theorem 1, since $\mathbf{F}(\mathbf{F} d e)(\mathbf{F e e})$ is a suitable h.c.i. of $\mathbf{F} c g$ and $\boldsymbol{F}(\mathbf{F} d e)(\mathbf{F} e e)$, we can substitute $\mathbf{F} d e$ for $c$ and combine the above two deductions to get

$$
\mathbf{F} b(\mathbf{F} d e) x, b y \vdash_{p} \mathbf{F} e e(E(x y)) .
$$

The result follows by Corollary 2.1 .

The construction of $B_{1}, \ldots, B_{4}$ is now complete, and only $C$ remains to be found. Define

Then

$$
C \equiv[u, x, y, z] . H x y u(y z)
$$

$$
\vdash_{p} \mathbf{F}(\mathbf{F} b b)(\mathbf{F}(\mathbf{F} c c)(\mathbf{F}(\mathbf{F} b c)(\mathbf{F} b c))) C .
$$

Proof. By (29) Corollary 1.4 used three times,

$$
\mathbf{F} a c x, \mathbf{F} b c y, \mathbf{F} b b u \vdash_{p} \mathbf{F} a a(H x y u) .
$$

Now

$\mathbf{F} d e y, d z \vdash_{p} e(y z)$. 
To combine these two deductions by the proof of Theorem 1 we need an h.c.i. of the sequences

$$
\langle\mathbf{F} b c, \mathbf{F} a a\rangle \quad\left\langle\mathbf{F} d e, F_{e g}\right\rangle .
$$

A suitable h.c.i. is $\langle\mathbf{F} b c, \mathbf{F} c c\rangle$, so substituting $c$ for $e$ and $a$, and $b$ for $d$ and combining the deductions gives

$\mathbf{F} c c x, \mathbf{F} b c y, \mathbf{F} b b u, b z \vdash_{p} c(H x y u(y z))$.

Corollary 2.1 then gives the result, completing the proof of Lemma 7 .

\section{REFERENCES}

1. H. B. Curry and R. Feys, Combinatory logic, North-Holland, Amsterdam, 1958.

2. H. B. Curry, Modified basic functionality in combinatory logic, Dialectica (to appear).

3. - On the definition of substitution, replacement and allied notions in an abstract formal system, Rev. Philos. de Louvain 50 (1952), 251-269.

4. J. A. Robinson, A machine-oriented logic based on the resolution principle, J. Assoc. Comput. Mach. 12 (1965), 23-41.

5. A. Grzegorczyk, Recursive objects in all finite types, Fund. Math. 54 (1964), 73-93.

University College of Swansea,

SWANSEA, WaLes 